The Astronomical Journal, 120:2190-2205, 2000 November

(C) 2000. The American Astronomical Society. All rights reserved. Printed in U.S.A.

\title{
CALTECH FAINT GALAXY REDSHIFT SURVEY. XIV. GALAXY MORPHOLOGY IN THE HUBBLE DEEP FIELD (NORTH) AND ITS FLANKING FIELDS TO $z=1.2^{1}$
}

\author{
Sidney van den Bergh, ${ }^{2}$ Judith G. Cohen, ${ }^{3}$ David W. Hogg, ${ }^{4,5,6}$ AND Roger Blandford ${ }^{4}$ \\ Received 2000 June 27; accepted 2000 August 2
}

\begin{abstract}
Morphological classifications are reported for Hubble Space Telescope images of 241 galaxies in the Hubble Deep Field and its flanking fields with measured redshifts in the interval $0.25<z<1.2$, drawn from a magnitude-limited redshift survey to $R=24.0$. The galaxies are divided into three groups with redshifts in the intervals $0.25-0.6,0.6-0.8$, and $0.8-1.2 . R_{606}$ images from the first group and $I_{814}$ images from the second and third groups are compared with $B$-band images of nearby galaxies. All classifications were therefore made at approximately the same rest wavelength. Selection biases are discussed. We corroborate and extend the results of earlier investigations by observing that

1. Most intermediate- and late-type galaxies with $z \gtrsim 0.5$ have morphologies that are dramatically different from those of local galaxies and cannot be shoehorned into the Hubble "tuning fork" classification scheme;

2. Grand-design spirals appear to be rare or absent for $z \gtrsim 0.3$;

3. Many $\mathrm{Sa}$ and $\mathrm{Sb}$ spirals with $z \gtrsim 0.6$ do not exhibit well-defined spiral arms, and the arms of distant Sc galaxies appear more chaotic than those of their nearby counterparts;

4. The fraction of all galaxies that are of types Sc and Scd drops from $23 \%$ at $z \sim 0$ to $5 \%$ for $z>0.6$.

5. Barred spirals are extremely rare for $z \gtrsim 0.5$;

6. Roughly one in five galaxies with $z \gtrsim 0.8$ is a compact objects that resembles local E, S0, or Sa galaxies.

7. Peculiar galaxies are more common beyond $z=0.3$, especially among late-type spirals, than they are at $z \sim 0$;

8. Merging galaxies, particularly those with three or more components, also become more common with increasing redshift.

On the basis of these and similar observations, it is inferred that the development of pronounced spiral structure was delayed until $\sim 5 \mathrm{Gyr}$ and that most bulges are probably not formed by disintegrating bars. Major morphological changes were still taking place only $\sim 5 \mathrm{Gyr}$ ago, even though changes in the integrated light of most galaxies were then much slower than they were $\sim 10 \mathrm{Gyr}$ ago.
\end{abstract}

Key words: galaxies: evolution - surveys

\section{INTRODUCTION}

The Hubble Space Telescope (HST) has, for the first time, allowed us to observe directly the evolution of galaxy morphology over a significant fraction of the age of the universe. Soon after the images of the Hubble Deep Field (HDF) and its flanking fields (FFs) became available (Williams et al. 1996; Ferguson, Dickinson, \& Williams 2000), it was clear that distant and young field galaxies were very different from their local, contemporary counterparts (Abraham et al. 1996a, 1996b). Indeed, half a lifetime of experience in galaxy classification (van den Bergh 1998) proved to be of only marginal usefulness in attempts to classify galaxies at

\footnotetext{
${ }^{1}$ Based in part on observations obtained at the W. M. Keck Observatory, which is operated jointly by the California Institute of Technology and the University of California.

${ }^{2}$ Dominion Astrophysical Observatory, Herzberg Institute of Astrophysics, National Research Council of Canada, 5071 West Saanich Road, Victoria, BC V9E 2E7, Canada; sidney.vandenbergh@nrc.ca.

${ }^{3}$ Palomar Observatory, Mail Stop 105-24, California Institute of Technology, Pasadena, CA 91125; jlc@astro.caltech.edu.

4 Theoretical Astrophysics, Mail Stop 130-33, California Institute of Technology, Pasadena, CA 91125; rdb@tapir.caltech.edu.

${ }^{5}$ Current address: Institute for Advanced Study, Olden Lane, Princeton, NJ 08540; hogg@ias.edu.

${ }^{6}$ Hubble Fellow.
}

intermediate and large look-back times. In the words of Martin Amis (1997), "You are living in a land you no longer recognise. You don't know the language anymore." More specifically, it was concluded that "The fraction of interacting and merging objects is seen to be significantly higher in the Hubble Deep Field than it is among nearby galaxies. Barred spirals are essentially absent from the deep sample. The fraction of early-type galaxies in the Hubble Deep Field is similar to the fraction of early-types in the Shapley-Ames Catalog, but the fraction of galaxies resembling archetypal grand-design late-type spiral galaxies is dramatically lower in the distant HDF sample" (van den Bergh et al. 1996). Because no redshifts were available, it was not possible to establish a timescale for galaxy evolution. It is the purpose of the present paper to reexamine these conclusions, using the HDF and FF images and the new redshift information (Cohen et al. 2000 and references therein), and to discuss the galaxy evolutionary timescale.

The initial reactions to the HDF images have been largely vindicated and significantly developed over the past 4 years (see Abraham 1999, 2000 for excellent reviews). The most complete information so far available on the morphological evolution of galaxies with $z \lesssim 1$ is by Brinchmann et al. (1998), who classified HST images of a complete sample of 341 galaxies, selected from both the Canada-France Redshift Survey (CFRS) and Low Dispersion Survey Spectro- 


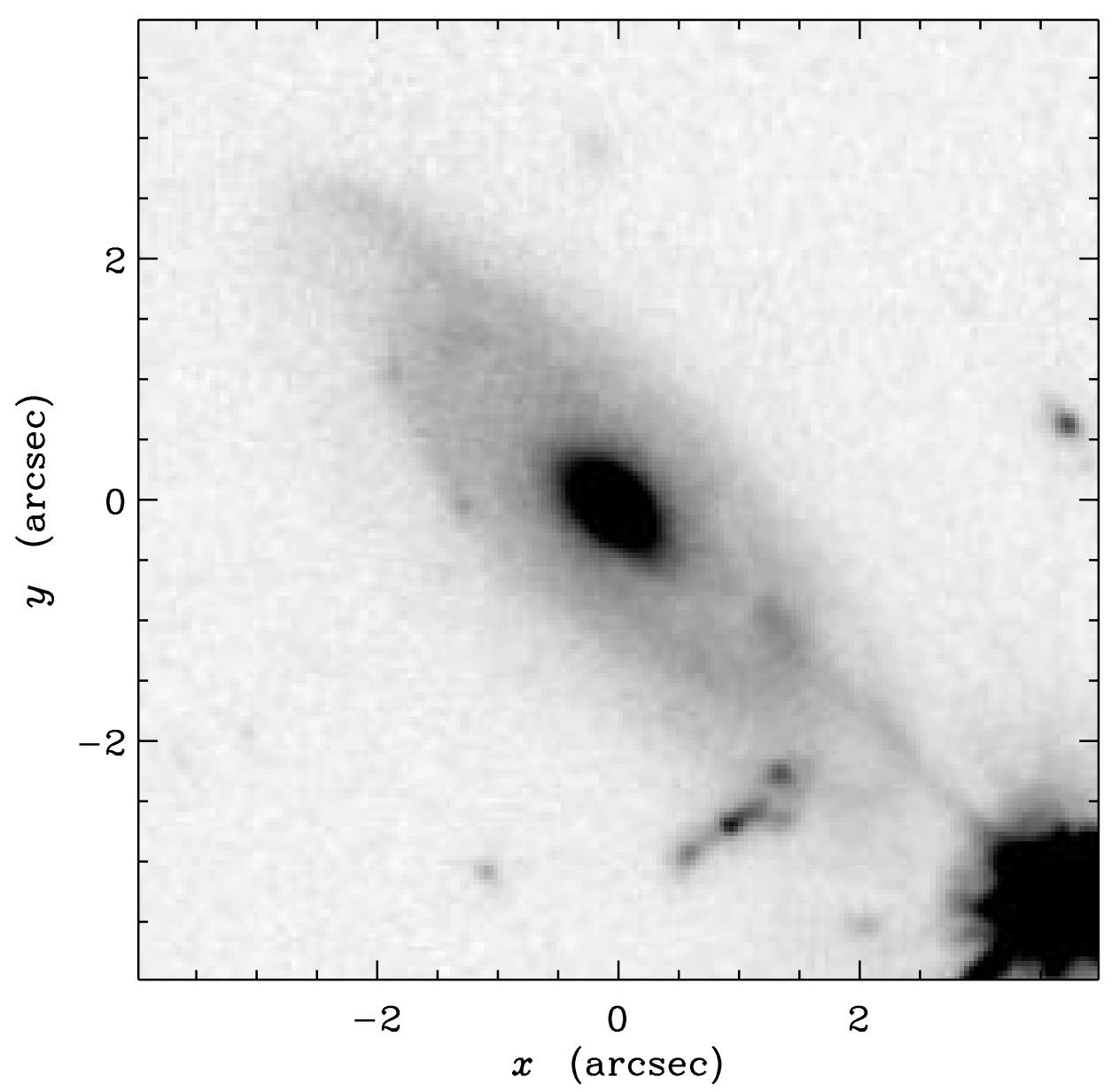

FIG. 1. - H36566_1245: An Sb spiral at $z=0.518$ that is peculiar because the spiral arms in the disk are underdeveloped compared with typical objects of similar type at $z \sim 0.0$. Note the multicomponent (background) merger below and to the right of this object. In this figure, as in all the others, an $8 \times 8$ arcsec ${ }^{2}$ section of the WFPC2 HST image is displayed in the orientation of the original HST image, whose scale is 0 ". 1 pixel $^{-1}$.

graph redshift survey (LDSS), for which ground-based redshifts were available. They found a substantial increase in the fraction of irregular galaxies from $\sim 9 \%$ at $z \sim 0.4$ to $\sim 32 \%$ at $z \sim 0.8$ and associated these galaxies with the increase in blue luminosity density with redshift. However, the reliability of this conclusion is undermined by the fact that the standard "irregular" galaxy that Brinchmann et al. used to calibrate their classifications appears to be an $\mathrm{Sb}$ pec galaxy with a central bulge and the kind of underdeveloped spiral structure that is typically seen in spirals at high redshift.

Using the same sample, Lilly et al. (1998) found that the sizes of large galaxy disks do not change significantly out to $z \sim 1$, but that the rate of star formation was elevated by a factor of 3 at $z \sim 0.7$. In a new approach, Abraham et al. (1999a) related the evolution of galaxy morphology to their internal star formation as monitored by their spatially resolved colors. They confirmed that spiral bulges predate their disks and followed different evolutionary histories. In a study that also included the HDF South, Abraham et al. (1999b) confirmed that barred spirals were comparatively rare prior to $z \sim 0.5$.

Elliptical galaxies, by contrast, appear to have evolved relatively little in density and luminosity since $z \sim 1$. There is evidence for ongoing and declining star formation although less than $5 \%$ of their stars are estimated to have formed over this time (Schade et al. 1999). They seem to have formed over an extended interval, $1 \lesssim z \lesssim 3$ (Odewahn et al. 1996; Driver et al. 1998). This is consistent with the work of Corbin et al. (2000), who find that 12 out of $111(11 \%)$ of their NICMOS images (in a data sample that extends to photometric redshifts as high as $z=2.7$ ) are probably elliptical galaxies.

Turning to irregular galaxies, the HST images of 285 CFRS/LDSS galaxies have been analyzed to derive the evolution of the merger fraction out to $z \sim 1$ (Le Fèvre et al. 2000). Up to $20 \%$ of luminous galaxies are found to be in physical pairs (some of which may be projections) at $z \sim 0.8$. A typical $L^{*}$ galaxy was found to have undergone about one to two mergers since $z \sim 1$. However, the "chain" galaxies, first identified by Cowie, $\mathrm{Hu}, \&$ Songaila (1995), appear to be neither edge-on spirals nor merger products (Abraham et al. 1999b).

In recent years, there has been a shift away from the traditional, descriptive, and, inevitably, somewhat subjective morphological approach toward more quantitative measures of galaxy structure such as scale lengths, central concentration, asymmetry, and so on. This is particularly valuable for connecting observations to increasingly sophisticated numerical simulations. However, galaxies are too variegated to be completely described by just a few numbers, and we believe that a simple morphological approach will continue to be of value, even at high redshift. After all, the durability of the original Hubble (1936, p. 45) 
TABLE 1

Redshifts and Morphological Classifications of Red Images of GalaXies With $0.25<z<0.60$

\begin{tabular}{|c|c|c|c|c|c|}
\hline $\mathrm{ID}^{\mathrm{a}}$ & Redshift $^{\mathbf{b}}$ & $\begin{array}{c}R^{\mathrm{c}} \\
(\mathrm{mag})\end{array}$ & $\begin{array}{c}\log L(B)^{\mathrm{d}} \\
(\mathrm{W})\end{array}$ & Classification & Comments \\
\hline F36427_1306 ...... & 0.485 & 22.02 & 36.10 & $\mathrm{Sab} / \mathrm{S} 0$ (edge-on) & Projected on merger remnant \\
\hline F36446_1304 ...... & 0.485 & 21.14 & 36.57 & $\mathrm{Sb}$ pec $+\mathrm{Ir} / \mathrm{pec}$ & Knots, but no arms, in disk ${ }^{\mathrm{e}}$ \\
\hline F36454_1325 ...... & 0.441 & 22.33 & 36.31 & Pec & High surface brightness \\
\hline F36458_1325 ...... & 0.321 & 20.71 & 36.23 & Sc pec & Only rudimentary spiral structure in disk \\
\hline F36563_1209 ...... & 0.321 & 23.22 & 35.34 & $\mathrm{Sb}$ pec & Knots, but on arms, in disk \\
\hline F36575_1212 ...... & 0.561 & 22.62 & 36.17 & Ir/merger & \\
\hline H36413_1141...... & 0.585 & 21.91 & 36.32 & $\mathrm{Sab}$ & Merging with H36414_1142f \\
\hline H36414_1142..... & 0.548 & 23.51 & 35.92 & Merger & At least three components ${ }^{f}$ \\
\hline H36416_1200...... & 0.483 & 25.03 & 35.16 & $\mathrm{~S} / \mathrm{Ir}$ & \\
\hline H36419_1205...... & 0.432 & 20.82 & 36.63 & $\mathrm{Sb}$ pec & Faint outer arms surrounding bright core with incipient arms \\
\hline H36429_1216...... & 0.454 & 20.51 & 36.79 & Sc pec & High surface brightness. Two chaotic arms \\
\hline H36439_1250..... & 0.557 & 20.84 & 36.89 & Sc pec & Knot plus incipient arm in disk \\
\hline H36442_1247...... & 0.555 & 21.40 & 36.64 & S pec & Protobulge but no arms (yet?) \\
\hline H36448_1200...... & 0.457 & 22.85 & 35.89 & S(B?)cd: & \\
\hline H36465_1203...... & 0.454 & 24.32 & 35.44 & Sa pec & Asymmetric + tidal debris \\
\hline H36465_1151..... & 0.503 & 22.00 & 36.42 & E1 & \\
\hline H36470_1236..... & 0.321 & 20.62 & 36.42 & S pec & E3-like core embedded in chaotic spiral (?) envelope \\
\hline H36472_1230...... & 0.421 & 22.63 & 35.91 & Sab pec & \\
\hline H36480_1309...... & 0.476 & 20.43 & 36.92 & Sa: & \\
\hline H36489_1245...... & 0.512 & 23.48 & 35.76 & $\mathrm{E}: 5$ pec & Asymmetric core embedded in fuzz \\
\hline H36493_1311..... & 0.477 & 21.97 & 36.30 & E1 & \\
\hline H36494_1316...... & 0.271 & 23.63 & 35.16 & Pec & \\
\hline H36496_1257...... & 0.475 & 21.91 & 36.31 & Sa pec & Asymmetric, multiple nuclei \\
\hline H36497_1313 ...... & 0.475 & 21.46 & 36.38 & $\mathrm{Sb}$ pec & Asymmetric \\
\hline H36501_1239...... & 0.474 & 20.43 & 36.87 & Pec & Has high surface brightness central region \\
\hline H36508_1251..... & 0.485 & 23.15 & 35.89 & $?$ & Disk containing multiple knots \\
\hline H36508_1255..... & 0.321 & 22.27 & 35.83 & S pec & Nucleus plus two knots in disk \\
\hline H36513_1420..... & 0.439 & 23.22 & 35.74 & $\mathrm{Sb}$ pec & Disk with no spiral arms. Off-center nucleus \\
\hline H36516_1220...... & 0.401 & 21.45 & 36.32 & Sab pec & \\
\hline H36517_1353...... & 0.557 & 21.08 & 36.80 & $\mathrm{Sb}$ pec/merger & Double nucleus \\
\hline H36519_1209...... & 0.458 & 22.75 & 35.94 & Pec & E1-like bulge embedded in asymmetric envelope \\
\hline H36519_1400..... & 0.559 & 23.03 & 36.00 & $\mathrm{Sb}$ pec (edge-on) & Off-center core \\
\hline H36526_1219...... & 0.401 & 23.11 & 35.72 & $\mathrm{E}: 4$ & \\
\hline H36528_1404...... & 0.498 & 23.45 & 35.82 & Pec/merger & \\
\hline H36534_1234...... & 0.560 & 22.78 & 36.11 & $\mathrm{Sb}$ pec & Off-center bulge \\
\hline H36536_1417...... & 0.517 & 23.36 & 35.82 & $\mathrm{Sb}$ pec (edge-on) & Nucleus + two knots in disk \\
\hline H36549_1314...... & 0.511 & 23.81 & 35.68 & Pec (edge-on) & Disk with nucleus + two knots \\
\hline H36551_1311...... & 0.321 & 23.58 & 35.33 & Pec & \\
\hline H36554_1402..... & 0.564 & 23.08 & 35.96 & Sbc pec & Off-center nucleus + one knot in disk \\
\hline H36555_1359...... & 0.559 & 23.74 & 35.73 & $\mathrm{Sb}$ (edge-on) & \\
\hline H36560_1329...... & 0.271 & 23.80 & 35.16 & $\mathrm{Ir} / \mathrm{pec}$ & \\
\hline H36566_1245...... & 0.518 & 20.06 & 37.11 & $\mathrm{Sb}$ pec & Bright E2-like bulge without spiral arms; see Fig. 1 \\
\hline H36569_1258...... & 0.520 & 23.84 & 35.76 & $\mathrm{Sa}$ & \\
\hline H36571_1225...... & 0.561 & 22.36 & 36.27 & Sc pec & Has one incipient spiral arm + three knots; see Fig. 2 \\
\hline H36572_1259..... & 0.475 & 21.07 & 36.61 & SB? pec & Bar or two knots \\
\hline H36580_1300...... & 0.320 & 22.04 & 35.89 & Merger & Two nuclei \\
\hline H36587_1252...... & 0.321 & 20.99 & 36.27 & SBbc pec & Probable bar in asymmetric two-armed spiral; see Fig. 3 \\
\hline H36594_1221...... & 0.472 & 23.53 & 35.67 & $\mathrm{~S} ?+\mathrm{Ir}:^{\mathrm{g}}$ & \\
\hline H37005_1234...... & 0.563 & 21.43 & 36.74 & S0/star & \\
\hline
\end{tabular}

${ }^{\text {a }}$ Names are Habcde fghi for objects in the HDF, where the object's $\mathbf{J} 2000.0$ coordinates are $12^{\mathrm{h}} a b^{\mathrm{m}} c d^{\mathrm{s}} e,+62^{\circ} f g^{\prime} h i^{\prime \prime}$. The initial letter is " $\mathrm{F}$ " for objects in the flanking fields.

${ }^{b}$ Redshifts are from Cohen et al. 2000.

${ }^{\mathrm{c}} R$ magnitudes are from Hogg et al. 2000.

${ }^{d}$ Rest-frame $B$ luminosities are from Cohen $2000 . M_{B}=-21.0$ (rest frame) $\equiv \log [L(B)(\mathrm{W})]=36.9$.

e The $\mathrm{Sb}$ pec is the object for which a redshift exists. The redshift of the fainter second galaxy is unknown.

${ }^{\mathrm{f}}$ The redshifts of this pair are not consistent with a merger, but the redshift of H36414_1142 is uncertain.

${ }^{\mathrm{g}}$ This is a close pair of faint galaxies separated by about $1^{\prime \prime}$. It is not clear which of them (or perhaps both) was included in the spectroscopic observations.

scheme for nearby galaxies is remarkable given all that we have learned about them since the 1930s.

In this paper we continue to use the traditional morphological classification, despite its manifest inadequacy at high redshift, for three reasons. The first is quite modest, to increase our confidence that galaxies at high redshift are quite different from local galaxies by enlarging the sample size. The second is to determine whether there are any counterparts to certain local galaxy types at high redshift. For example, if it is believed on dynamical grounds (see $\S 4.1$ ) that grand-design spirals take a minimum of $\sim 10 \mathrm{Gyr}$ to grow, then the discovery of just one bona fide example at 
TABLE 2

Redshifts AND MoRPhological Classifications of INFraRed Images of GalaXies with $0.60<z<0.80$

\begin{tabular}{|c|c|c|c|c|c|}
\hline $\mathrm{ID}^{\mathrm{a}}$ & Redshift $^{\text {b }}$ & $\begin{array}{c}R^{\mathrm{c}} \\
(\mathrm{mag})\end{array}$ & $\begin{array}{c}\log L(B)^{\mathrm{d}} \\
(\mathrm{W})\end{array}$ & Classification & Comments \\
\hline F36194_1428 ...... & 0.798 & 22.60 & 36.61 & Ir? & Multiple nuclei \\
\hline F36244_1454 ...... & 0.628 & 20.34 & 37.34 & E2 & \\
\hline F36243_1525 ...... & 0.682 & 22.78 & 36.48 & $\mathrm{E}: 3$ pec & Asymmetric \\
\hline F36247_1510 ..... & 0.641 & 20.41 & 37.26 & $\mathrm{Sa}$ & \\
\hline F36249_1252 ..... & 0.631 & 22.76 & 36.22 & Ir (edge-on) & \\
\hline F36250_1341 ...... & 0.654 & 24.32 & 35.73 & $\mathrm{Star} / \mathrm{E} 0$ & \\
\hline F36254_1519 ...... & 0.642 & 21.82 & 36.60 & $\mathrm{Sb}$ pec & Slightly asymmetric \\
\hline F36270_1509 ...... & 0.794 & 21.60 & 37.36 & $\mathrm{Sa}$ & \\
\hline F36275_1418 ...... & 0.751 & 22.37 & 36.54 & $\mathrm{~S}$ (edge-on) & \\
\hline F36284_1037 ...... & 0.760 & 22.76 & 36.39 & E0 & \\
\hline F36287_1357 ...... & 0.639 & 23.05 & 36.10 & $\mathrm{Sb}$ pec? & \\
\hline F36290_1346 ...... & 0.693 & 23.02 & 36.25 & $\mathrm{E}: 2$ & Compact \\
\hline F36297_1324 ...... & 0.758 & 23.25 & 36.23 & Pec/merger? & Member of a compact group \\
\hline F36297_1329 ...... & 0.748 & 23.03 & 36.44 & Pec & Member of a compact group \\
\hline F36299_1403 ...... & 0.793 & 21.97 & 36.91 & Merger? & \\
\hline F36334_1432 ...... & 0.748 & 23.08 & 36.31 & $\mathrm{~S}:$ pec & In compact group \\
\hline F36340_1054 ...... & 0.762 & 21.55 & 36.87 & Ir or protogalaxy & In a group \\
\hline F36362_1319 ...... & 0.680 & 22.20 & 36.44 & Lumpy protogalaxy? & \\
\hline F36370_1159 ...... & 0.779 & 21.56 & 36.84 & Proto-Sc? & \\
\hline F36379_0922 ...... & 0.767 & 21.43 & 37.08 & $\mathrm{Sb}:$ pec & Has two "nuclei," not a bar! \\
\hline F36384_1312 ...... & 0.635 & 22.27 & 36.38 & $\mathrm{Sb}$ pec & \\
\hline F36390_1006 ...... & 0.635 & 21.22 & 36.81 & $?$ & Has double core \\
\hline F36405_1003 ...... & 0.749 & 22.39 & 36.58 & Sc: & \\
\hline F36415_0902 ...... & 0.713 & 22.31 & 36.59 & $?$ & \\
\hline F36427_1503 ...... & 0.698 & 23.17 & 36.18 & Pec & \\
\hline F36454_1523 ...... & 0.683 & 22.06 & 36.57 & S: & \\
\hline F36481_1102 ...... & 0.650 & 22.58 & 36.40 & $\mathrm{Sb}$ & \\
\hline F36481_1002 ...... & 0.682 & 21.92 & 36.60 & $\mathrm{Ir} / \mathrm{pec}$ & \\
\hline F36499_1058 ...... & 0.684 & 22.63 & 36.36 & $\mathrm{Sa}:$ & \\
\hline F36575_1210 ...... & 0.665 & 21.10 & 36.98 & $\mathrm{E}: 0+\mathrm{Sb}:$ & Second galaxy is $\mathrm{F} 36575 \_1211^{\mathrm{e}}$ \\
\hline F36580_1137 ...... & 0.681 & 23.00 & 36.17 & $\mathrm{E}: 1$ & \\
\hline F36588_1434 ...... & 0.678 & 20.85 & 37.14 & $\mathrm{~S}$ pec & \\
\hline F36598_1449 ...... & 0.762 & 21.62 & 37.04 & $\mathrm{~S}+\mathrm{Sb}$ & Merger \\
\hline F37015_1129 ...... & 0.779 & 21.45 & 37.07 & Merger & Has three nuclei. In group \\
\hline F37017_1144 ...... & 0.744 & 22.10 & 36.71 & $\mathrm{Sa}:$ & Multiple nuclei? \\
\hline F37020_1517 ...... & 0.744 & 23.56 & 36.24 & Sab: & \\
\hline F37036_1353 ...... & 0.745 & 21.63 & 36.78 & $\mathrm{Sb}$ pec & \\
\hline F37058_1317 ...... & 0.753 & 21.95 & 36.74 & Sc: & \\
\hline F37061_1332 ...... & 0.753 & 21.85 & 36.83 & Sbc pec & Multiple nuclei? \\
\hline F37069_1208 ...... & 0.693 & 24.13 & 35.78 & $\mathrm{E}: 0$ & \\
\hline F37072_1214 ..... & 0.655 & 22.19 & 36.62 & $\mathrm{Sb}$ & \\
\hline F37074_1356 ...... & 0.752 & 23.65 & 36.19 & $\mathrm{Sa}$ & \\
\hline F37080_1246 ...... & 0.654 & 21.80 & 36.64 & $\mathrm{Sb}$ & \\
\hline F37083_1320 ..... & 0.785 & 22.86 & 36.44 & Pec & Tadpole-like \\
\hline F37088_1117 ...... & 0.639 & 23.05 & 36.18 & $\mathrm{Sb}$ pec & Asymmetric \\
\hline F37088_1214 ...... & 0.788 & 23.90 & 36.05 & $\mathrm{Sa}$ & \\
\hline F37105_1141 ...... & 0.789 & 21.20 & 37.10 & $\mathrm{Sb}$ pec $+\mathrm{S}$ & \\
\hline F37107_1431 ...... & 0.677 & 22.39 & 36.61 & $\mathrm{E} 1$ & \\
\hline F37108_1059 ...... & 0.747 & 24.25 & 36.05 & $?+?$ & Low surface brightness \\
\hline F37113_1545 ...... & 0.692 & 22.43 & 36.42 & $\mathrm{dIr} ?$ & Low surface brightness \\
\hline F37115_1042 ...... & 0.778 & 21.97 & 36.74 & Sbc pec & \\
\hline F37163_1432 ...... & 0.635 & 22.50 & 36.30 & $\mathrm{Sb}$ pec & Asymmetric \\
\hline F37192_1143 ...... & 0.784 & 22.81 & 36.47 & $\mathrm{Sb}$ pec & \\
\hline F37213_1120 ...... & 0.656 & 22.22 & 36.47 & Sc pec & \\
\hline H36389_1219..... & 0.609 & 22.14 & 36.43 & Pec & High surface brightness. Complex core $=$ merger $?$ \\
\hline H36436_1218...... & 0.752 & 22.56 & 36.46 & E1 & \\
\hline H36438_1142...... & 0.765 & 21.26 & 37.32 & $\mathrm{E} 1$ & In group \\
\hline H36459_1201 ...... & 0.679 & 23.88 & 35.85 & Pec & High surface brightness. Has two cores \\
\hline H36470_1213..... & 0.677 & 24.63 & 35.51 & Pec & Core + asymmetric fuzz \\
\hline H36471_1414...... & 0.609 & 23.92 & 35.82 & E5 & \\
\hline H36487_1318..... & 0.753 & 22.87 & 36.40 & Sc pec & Very lumpy structure. In cluster \\
\hline H36494_1406...... & 0.752 & 21.95 & 36.83 & E3 & \\
\hline H36498_1242 ...... & 0.751 & 24.38 & 35.64 & dIr (edge-on) & In cluster \\
\hline H36502_1245...... & 0.680 & 21.74 & 36.86 & E3 & In cluster \\
\hline
\end{tabular}


TABLE 2-Continued

\begin{tabular}{|c|c|c|c|c|c|}
\hline $\mathrm{ID}^{\mathrm{a}}$ & Redshift $^{\mathrm{b}}$ & $\begin{array}{c}R^{\mathrm{c}} \\
(\mathrm{mag})\end{array}$ & $\begin{array}{c}\log L(B)^{\mathrm{d}} \\
(\mathrm{W})\end{array}$ & Classification & Comments \\
\hline H36538_1254...... & 0.642 & 20.95 & 37.01 & S pec & High surface brightness \\
\hline H36555_1245...... & 0.790 & 23.08 & 36.79 & $\mathrm{~S}$ & \\
\hline H36586_1221 ...... & 0.682 & 23.40 & 36.03 & E2 & \\
\hline F37222_1124 ...... & 0.786 & 22.33 & 36.71 & $\mathrm{Sa}$ & \\
\hline
\end{tabular}

${ }^{\mathrm{a}}$ Names are Habcde fghi for objects in the HDF, where the object's J2000.0 coordinates are $12^{\mathrm{h}} a b^{\mathrm{m}} c d^{\mathrm{s}} \cdot e,+62^{\circ} f g^{\prime} h i^{\prime \prime}$. The initial letter is " $F$ " for objects in the flanking fields.

${ }^{\mathrm{b}}$ Redshifts are from Cohen et al. 2000

${ }^{\mathrm{c}} R$ magnitudes are from Hogg et al. 2000.

${ }^{d}$ Rest-frame $B$ luminosities are from Cohen $2000 . M_{B}=-21.0$ (rest frame) $\equiv \log [L(B)(\mathrm{W})]=36.9$.

e The redshifts of the two galaxies are both known and not consistent with a merger.

$z \sim 1$ would be highly significant. The third reason is that it is important to determine the chronology of these changes, and in the absence of an adequate high-redshift classification, the best approach is to compare with the wellunderstood local classification scheme and to discover when it starts to fail.

In the following section, we describe our procedure and discuss some possible selection biases. In $\S 3$ we give our empirical conclusions, and we conclude with a brief interpretation of their implications for physical theories of galaxy formation.

\section{GALAXY CLASSIFICATION}

\subsection{Morphology Sample}

Our sample is based on the redshift survey in the region of the HDF by Cohen et al. (2000), which includes 671 objects. This survey is $92 \%$ complete with respect to the photometric catalogs of Hogg et al. (2000) to $R \leq 24$ in the HDF itself, and also $92 \%$ complete to $R \leq 23$ in a region $8^{\prime}$ in diameter centered on the HDF. See Cohen et al. (2000) for details of the samples and the number of stars, galaxies, and high-redshift $(z>1.5)$ galaxies, AGNs, and QSOs in the HDF and in the flanking fields. ${ }^{7}$

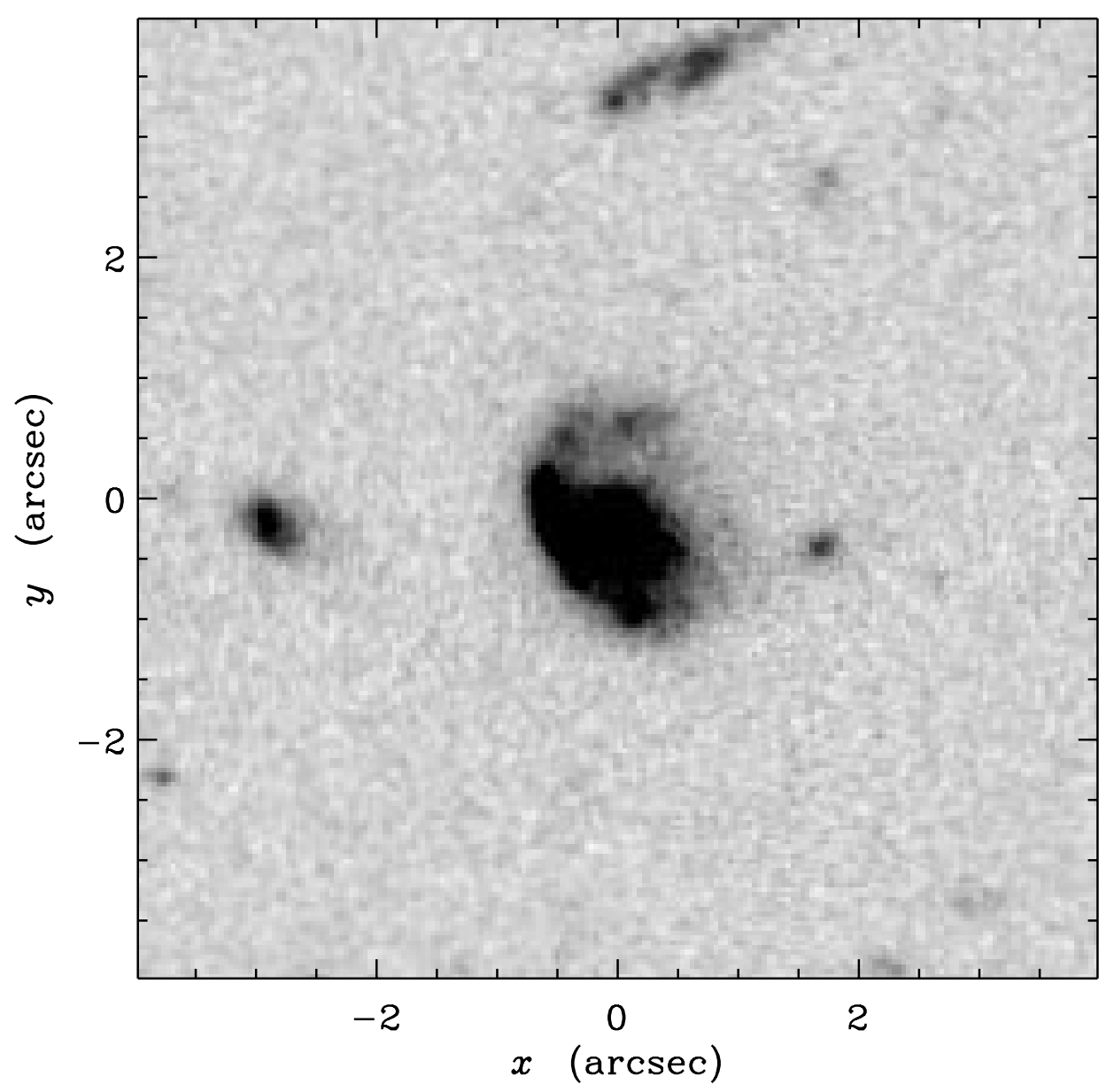

FIG. 2.-H36571_1225: Note the malformed single spiral arm of this peculiar Sc galaxy at $z=0.561$. 


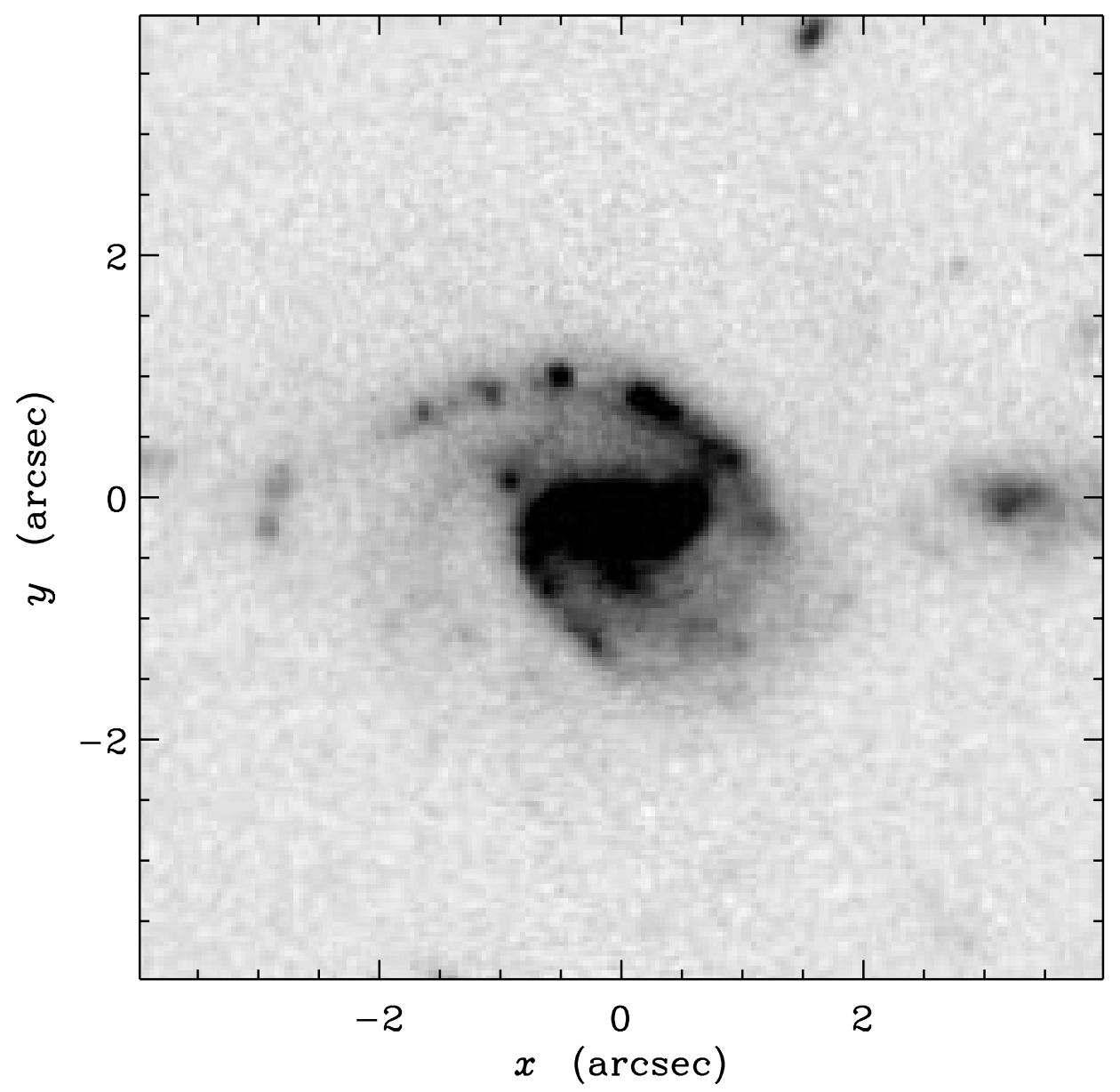

FIG. 3.-H36587_1252: The most distant "certain" barred spiral in the present sample (at $z=0.321)$. Note the difference in the lengths of spiral arms in this $\mathrm{SBc}$ pec galaxy.

The sample used for morphology and discussed here is divided into three redshift intervals, $0.25<z<0.60$, $0.60<z<0.80$, and $0.80<z<1.20$. In each interval, the sample contains all available galaxies in the spectroscopic redshift catalog with suitable HST images. Thus the lowredshift group contains 49 galaxies, of which 43 are drawn from the HDF. Four galaxies are in the Planetary Camera field of the WFPC2 image, and two others are just outside the boundary adopted for the HDF but within the coverage of the HST $R$ image. (It is unfortunate that there is no $R$ HST image of the rest of the area of the FFs.) Apart from this, there is no bias in magnitude, color, or location in this subsample. The intermediate-redshift group includes 70 galaxies, while the high-redshift group includes 120 galaxies. Note that the WFPC2 HST $I_{814}$ images do not cover the entire area of the flanking fields, and hence some galaxies within the area of the redshift survey could not be used. The fraction of galaxies drawn from the HDF and from the FFs is as would be expected based on the relative areas and the rise in galaxy counts as a function of $R$ magnitude in this magnitude regime.

\footnotetext{
${ }^{7}$ The assembly of the sample for the present morphological study was carried out before the final version of Cohen et al. (2000) was available. A few galaxies $(\sim 10)$ whose redshifts were only determined in the late fall of 1999 that are included in Cohen et al. (2000) are not included here.
}

\subsection{Biases and Selection Effects}

From a practical point of view, the morphological classification of individual galaxies at $z \sim 1$ presents three distinct challenges beyond similar classifications of nearby systems. The first is that the images used for the classification of nearby galaxies might contain as many as $\sim 10,000$ pixels, whereas those of very distant $H S T$ images may contain only $\sim 100$ pixels, and apparent changes in fine-scale features may simply be a consequence of resolution. Furthermore, as a result of longer exposure times, the quality of the HDF images is markedly superior to that of the FF images. This may lead to some misinterpretation of the morphologies of the faintest FF galaxies, especially the most compact examples. For example, it is often not possible to distinguish Galactic stars with certainty from compact ellipticals of Hubble types E0 and E1. (All of the objects in the present sample, including those that have morphological classification "star" or "E0/star" are extragalactic, on the basis of their redshifts, though.) The comparative statements below are restricted to those that can be made with confidence on the basis of the poorest images. (We note, in passing, that it is the large dynamic range of CCD detectors that makes them particularly suitable for use in galaxy classification; van den Bergh \& Pierce 1990.)

The second challenge is to correct for band shifting. Galaxy classification is traditionally performed in the $B$ 


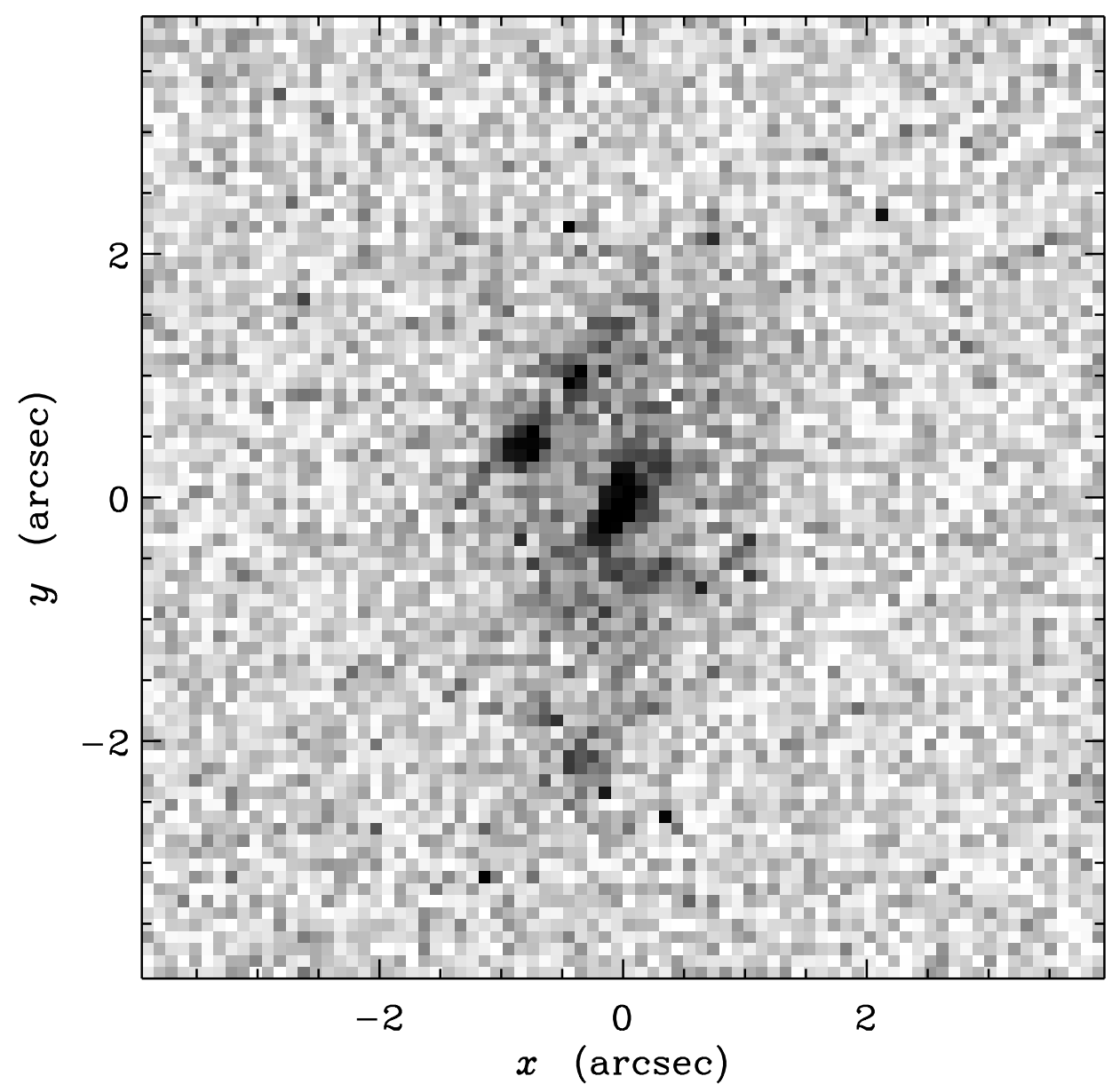

FIG. 4.-F36287_1-23: Example at $z=0.936$ of merger of relatively low surface brightness galaxies that may eventually evolve into an Sc galaxy.

$(440 \mathrm{~nm})$ band. We find that galaxy morphology varies sufficiently slowly with wavelength (see also Fig. 3 of Ferguson et al. 2000) that it is adequate to compare $R_{606}$ images from the $0.25-0.60$ redshift interval (the shift is exact for $z=0.38$ ) and $I_{814}$ for $0.60<z<1.20$ (exact for $z=0.85$ ) with local samples. In particular, the $z$-dependence of the frequency of barred spirals ( 3.2 ) cannot be attributed to band shifting, as has sometimes been suggested (e.g., Bunker et al. 2000; Eskridge et al. 2000). Some small band-shift effects could, however, still be experienced for galaxies that have $1.0 \lesssim$ $z \lesssim 1.2$. In such objects the core-to-halo ratio might be depressed, and giant stellar associations may appear slightly enhanced, relative to similar structures seen at lower redshifts.

The third challenge is that, in a magnitude-limited survey, we are comparing more luminous objects at high redshift with less luminous objects at low $z$. We emphasize that our local comparison sample is not the HDF but the more extensive Shapley-Ames catalog. With respect to the sample of the Revised Shapley-Ames Catalog (RSA), the median $M_{B}$ (rest frame) for the HDF sample is about $0.3 \mathrm{mag}$ fainter than that of the RSA, where the median for the RSA sample is taken from Figure 5 of Sandage \& Tammann (1981) and has been adjusted to our adopted value of $H_{0}$ (only RSA galaxies with known redshifts as of that date are plotted in this figure). A correction for internal absorption was applied to the late-type spirals in the RSA sample, while none has been applied here. Hence the two samples are comparable in their luminosity range; we are in fact seeing quite far below $L^{*}$ in the HDF sample.

Relative to the median galaxy in $R$ at $z=0.3$, the absolute magnitudes of the median galaxies with $z=0.6,0.8$, and 1.2 are respectively $-1.8,-2.6$, and -3.6 smaller solely as a result of the larger distance using our adopted cosmology. ${ }^{8}$ The median (in $z$ ) galaxies in the intermediateand high-redshift intervals have absolute magnitudes -1.1 and -1.9 smaller than the median in the low- $z$ interval. It can be argued that about half of this luminosity selection is appropriate because it compensates passive stellar evolutionary effects. However, taking a strictly morphological approach, we have ignored this and simply make comparative observations about galaxies with similar luminosities to those at high redshift. Our prime conclusions are quite robust to this choice. However, luminosity selection is a serious concern when probing the evolution of secondary characteristics such as disk surface brightness (Simard et al. 1999).

\subsection{Sampling}

An additional and related caveat concerns the size of the sample and the sensitivity to fluctuations resulting from the luminosity selection. A good measure of this is the number

\footnotetext{
${ }^{8}$ In this paper, we assume a flat universe with $\Omega_{M}=0.3, h=0.7$, and age $t_{0}=14 \mathrm{Gyr}$.
} 
TABLE 3

Redshifts aNd Morphological Classifications of INFraRed Images of GalaXies with $0.80<z<1.20$

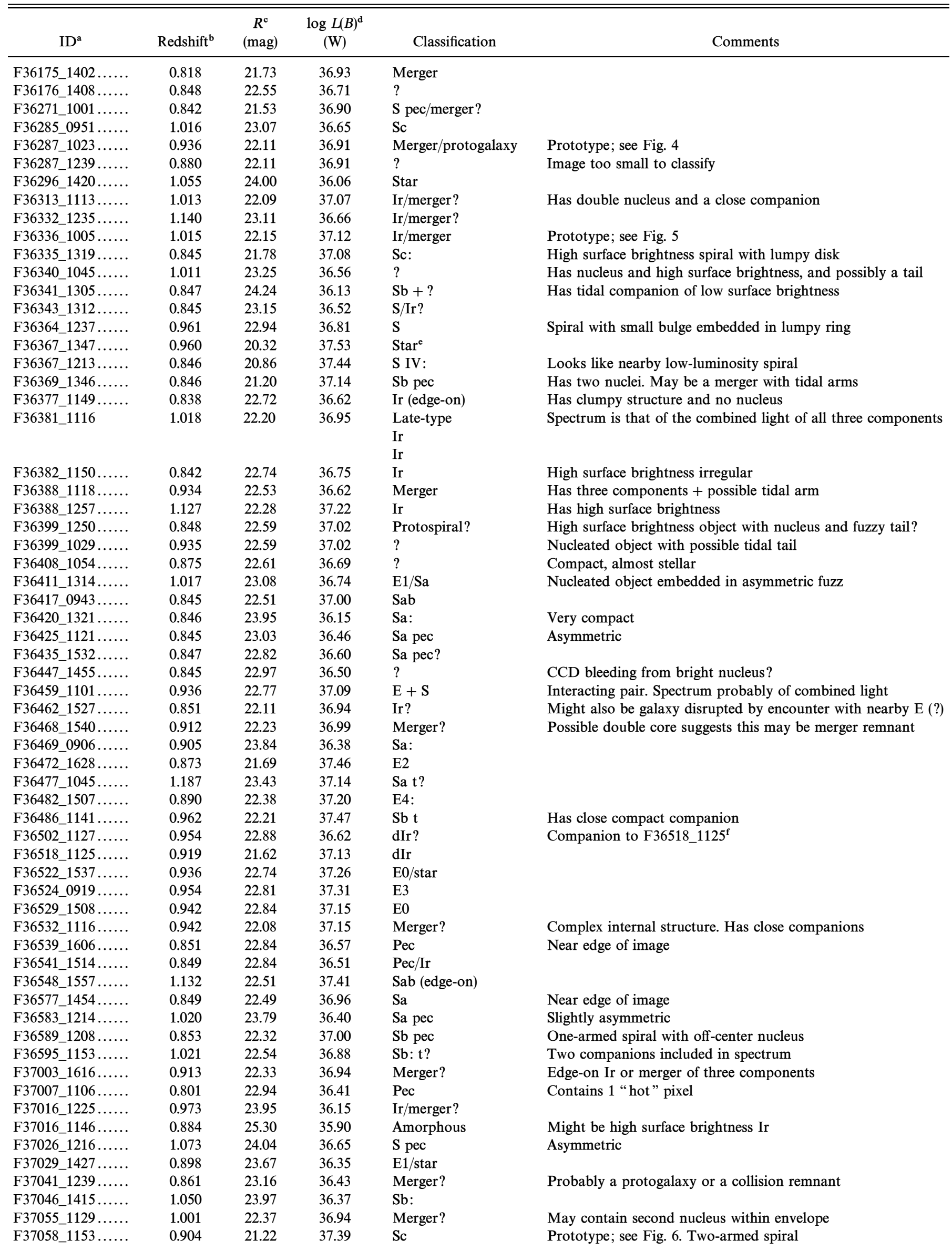


TABLE 3-Continued

\begin{tabular}{|c|c|c|c|c|c|}
\hline $\mathrm{ID}^{\mathrm{a}}$ & Redshift $^{\mathrm{b}}$ & $\begin{array}{c}R^{\mathrm{c}} \\
(\mathrm{mag})\end{array}$ & $\begin{array}{l}\log L(B)^{\mathrm{d}} \\
\text { (W) }\end{array}$ & Classification & Comments \\
\hline F37058_1423. & 0.970 & 22.48 & 37.10 & $?$ & Strongly nucleated \\
\hline F37065_1512 & 0.840 & 22.94 & 36.48 & $\begin{array}{l}\text { E0/star } \\
\text { S/Ir }\end{array}$ & Redshift refers to combined light of A and B \\
\hline F37078_1605 ...... & 0.936 & 21.88 & 37.27 & Sc & Clumps in disk/arms \\
\hline F37083_1252 ...... & 0.838 & 22.20 & 36.85 & $\mathrm{Sb}$ & \\
\hline F37083_1514 ...... & 0.839 & 21.62 & 37.10 & $\mathrm{Sb}$ pec & Asymmetric bar? \\
\hline F37086_1128 ...... & 0.907 & 22.23 & 36.76 & Merger & At least two components, one of these is distorted \\
\hline F37089_1202 ..... & 0.855 & 22.90 & 36.52 & $\mathrm{Sc}$ & Single nucleus embedded in clumpy envelope \\
\hline F37096_1055 ..... & 0.858 & 23.16 & 36.40 & Ir & Ir or high surface brightness merger \\
\hline F37114_1055 ...... & 0.855 & 22.44 & 36.73 & S pec & Asymmetric \\
\hline F37126_1546 ...... & 0.937 & 22.08 & 37.07 & E1 & \\
\hline F37129_1028 ..... & 0.858 & 22.62 & 36.73 & $\mathrm{Ir} /$ merger & Edge-on Ir, or two-component merger \\
\hline F37133_1054 ..... & 0.936 & 21.87 & 36.99 & Sa t? & \\
\hline F37141_1044 ...... & 0.821 & 22.32 & 36.70 & E1 & \\
\hline F37143_1221 ..... & 1.084 & 24.12 & 36.66 & $\mathrm{Sa}$ & \\
\hline F37154_1212 ..... & 1.014 & 23.25 & 37.06 & $?$ & Compact, asymmetric \\
\hline F37159_1213 ..... & 1.020 & 23.27 & 36.86 & $\mathrm{Sb}:$ & Core embedded in extended (tidal?) envelope \\
\hline F37167_1042 ..... & 0.821 & 21.59 & 36.97 & Merger & Merger prototype; see Fig. 7 . Three or four components \\
\hline F37180_1248 ...... & 0.912 & 22.89 & 36.78 & $\mathrm{Sb}:$ & \\
\hline F37196_1256 ..... & 0.909 & 23.31 & 36.67 & $\mathrm{Sa} / \mathrm{E} 4$ & \\
\hline F37221_1210 ...... & 0.928 & 23.97 & 36.60 & Merger & Prototypical merger; see Fig. 8. At least four components \\
\hline F37224_1216 ...... & 0.963 & 22.23 & 37.09 & $\mathrm{St}$ & One of the components of F37221_1210 \\
\hline H36384_1231..... & 0.944 & 22.87 & 36.87 & $\mathrm{~S}$ & Edge-on \\
\hline H36386_1233..... & 0.904 & 24.04 & 36.18 & Sab & Edge-on \\
\hline H36396_1230..... & 0.943 & 24.40 & 35.79 & $\mathrm{~S}+\mathrm{E} 2:$ & Merger \\
\hline H36400_1207...... & 1.015 & 22.75 & 37.17 & Star & \\
\hline H36408_1203..... & 1.010 & 23.49 & 36.50 & $\mathrm{Pec} / \mathrm{Ir}$ & Distorted edge-on irregular? \\
\hline H36408_1205..... & 0.882 & 22.94 & 36.63 & Star + galaxy & \\
\hline H36431_1242...... & 0.849 & 22.34 & 37.11 & E2 & \\
\hline H36432_1148..... & 1.010 & 23.10 & 37.01 & $\mathrm{Sb}$ & High surface brightness two-armed spiral \\
\hline Н36441_1240...... & 0.875 & 23.39 & 36.36 & Merger & \\
\hline H36443_1133..... & 1.050 & 21.96 & 37.76 & E1 & \\
\hline H36444_1142..... & 1.020 & 24.30 & 36.68 & Merger & Prototype; see Fig. 9. Edge-on \\
\hline Н36461_1246...... & 0.900 & 22.86 & 36.92 & E1 & \\
\hline H36461_1142...... & 1.013 & 21.52 & 37.39 & Sab pec & \\
\hline H36463_1404_..... & 0.962 & 21.69 & 37.41 & Sa pec & \\
\hline H36467_1144..... & 1.060 & 24.23 & 36.55 & Pec & \\
\hline H36477_1232..... & 0.960 & 23.80 & 36.48 & E3 & \\
\hline H36483_1214 ..... & 0.962 & 23.87 & 36.75 & $\mathrm{~S}(\mathrm{~B}) \mathrm{bc} \mathrm{t}$ & Barred, or tidally distorted, spiral \\
\hline H36486_1328..... & 0.958 & 23.14 & 36.68 & Merger & Has three or four components \\
\hline H36490_1221...... & 0.953 & 22.59 & 36.81 & Merger? & High surface brightness image has core and four outer lumps \\
\hline H36492_1148..... & 0.961 & 23.26 & 36.69 & $\mathrm{E} 4 / \mathrm{Sa}$ & \\
\hline Н36493_1155...... & 0.961 & 23.36 & 36.40 & $\mathrm{Star} / \mathrm{E} 0$ & \\
\hline H36503_1418..... & 0.819 & 23.41 & 36.38 & S pec & Nuclear bulge embedded in eccentric ring \\
\hline H36504_1315..... & 0.851 & 23.41 & 36.38 & $\mathrm{~S}$ pec & Has distorted disk \\
\hline Н36519_1332..... & 1.087 & 23.59 & 36.76 & E1/star & \\
\hline Н36540_1354 ...... & 0.851 & 22.72 & 36.70 & $\mathrm{~S} / \mathrm{E} 3 \mathrm{pec}$ & Has distorted core \\
\hline H36551_1303...... & 0.952 & 24.29 & 36.51 & E3 & High surface brightness \\
\hline Н36553_1311..... & 0.968 & 22.86 & 37.11 & $\mathrm{E} / \mathrm{Sa}:$ & High surface brightness \\
\hline Н36555_1353..... & 1.147 & 22.85 & 36.90 & Sc/Ir: & \\
\hline H36555_1249..... & 0.950 & 23.53 & 36.48 & Protospiral & See Fig. 10 \\
\hline Н36566_1220..... & 0.930 & 23.15 & 36.87 & $\mathrm{Sa}$ & High surface brightness. Has companion \\
\hline H36576_1315..... & 0.952 & 22.94 & 36.62 & $\mathrm{~S} / \mathrm{Ir}$ & \\
\hline
\end{tabular}

${ }^{\text {a }}$ Names are Habcde fghi for objects in the HDF, where the object's J2000.0 coordinates are $12^{\mathrm{h}} a b^{\mathrm{m}} c d^{\mathrm{s}} e,+62^{\circ} f g^{\prime} h i^{\prime \prime}$. The initial letter is " $\mathrm{F}$ " for objects in the flanking fields.

b Redshifts are from Cohen et al. 2000.

${ }^{\mathrm{c}} R$ magnitudes are from Hogg et al. 2000.

d Rest frame $B$ luminosities are from Cohen 2000. $M_{B}=-21.0$ (rest frame) $\equiv \log [L(B)(\mathrm{W})]=36.9$.

e This object is a QSO from Keck spectroscopy.

${ }^{\mathrm{f}}$ These two objects have rather discrepant well-determined redshifts and are probably not physically associated.

density of $L^{*}$ galaxies, specifically $\Phi^{*} V_{c}$, where $V_{c}$ is the comoving volume and $L^{*}$ and $\Phi^{*}$ are determined locally, expected in our three redshift intervals (cf. Ferguson et al. 2000). This corresponds to 8,10 , and 20 bright galaxies on the HDF and 9 times as many galaxies in the FFs. However, galaxies are strongly clustered and the fluctuations over small comoving volumes are much larger than Poissonian. Furthermore, the HDF was carefully selected 
TABLE 4

Morphological Types of Nearby and Distant Clusters

\begin{tabular}{|c|c|c|c|c|}
\hline \multirow[b]{2}{*}{ GalaXy Type } & \multirow{2}{*}{$\begin{array}{c}\text { SHAPLEY-AMES } \\
z \sim 0 \\
(\%)\end{array}$} & \multicolumn{3}{|c|}{ HDF + FLANKING FIELDS (\%) } \\
\hline & & $0.25-0.60$ & $0.60-0.80$ & $0.80-1.20$ \\
\hline $\mathrm{E}+\mathrm{S} 0+\mathrm{E} / \mathrm{S} 0 \ldots$ & 22 & 11 & 21 & 16 \\
\hline $\mathrm{E} / \mathrm{Sa}+\mathrm{S} 0 / \mathrm{Sa} \ldots \ldots \ldots \ldots$ & 1 & 0 & 0 & 2 \\
\hline $\mathrm{Sa}+\mathrm{Sab} \ldots \ldots \ldots \ldots \ldots$ & 7 & 15 & 11 & 13 \\
\hline $\mathrm{Sb}+\mathrm{Sbc} \ldots \ldots \ldots \ldots \ldots \ldots$ & 27 & 26 & 23 & 10 \\
\hline $\mathrm{Sc}+\mathrm{Sc} / \mathrm{Ir}+\mathrm{Scd} \ldots \ldots \ldots$ & 23 & 10 & 5 & 5 \\
\hline Ir $\ldots \ldots \ldots \ldots \ldots \ldots \ldots \ldots$ & 2 & 5 & 7 & 12 \\
\hline $\mathrm{S}$....................... & 10 & 11 & 10 & 11 \\
\hline Pec, ?, protogalaxy ...... & 7 & 16 & 19 & 12 \\
\hline Merger ................... & $1^{\mathrm{a}}$ & 7 & 4 & 15 \\
\hline Total in sample ........ & 936 & 50 & 70 & $120^{\mathrm{b}}$ \\
\hline
\end{tabular}

NoTE.-Because of rounding errors, percentages do not all add to 100 .

${ }^{a}$ From Appendix to van den Bergh 1960c.

"Includes four extragalactic objects classified as "star" and one classified as "amorphous."

to avoid bright galaxies (Ferguson et al. 2000), and there is a clear deficit of these for $z \lesssim 0.3$ (Cohen et al. 2000). In particular, the nature of the galactic population in the fields studied in the present investigation might have been affected by the vagaries of the density-morphology relation (Dressler 1980) along the line of sight. This concern dictated our choice of $z=0.25$ as the lower limit of our redshift intervals.

\subsection{Morphological Typing}

The morphological classifications reported in this paper were made by S. v. d. B. and are on the DDO system of van den Bergh (1960a, 1960b, 1960c). The images were supplied to him as $20 \times 20 \operatorname{arcsec}^{2}$ thumbnails along with the redshift interval and no other information. The areal scale on the sky per pixel was the same for all the images. All images

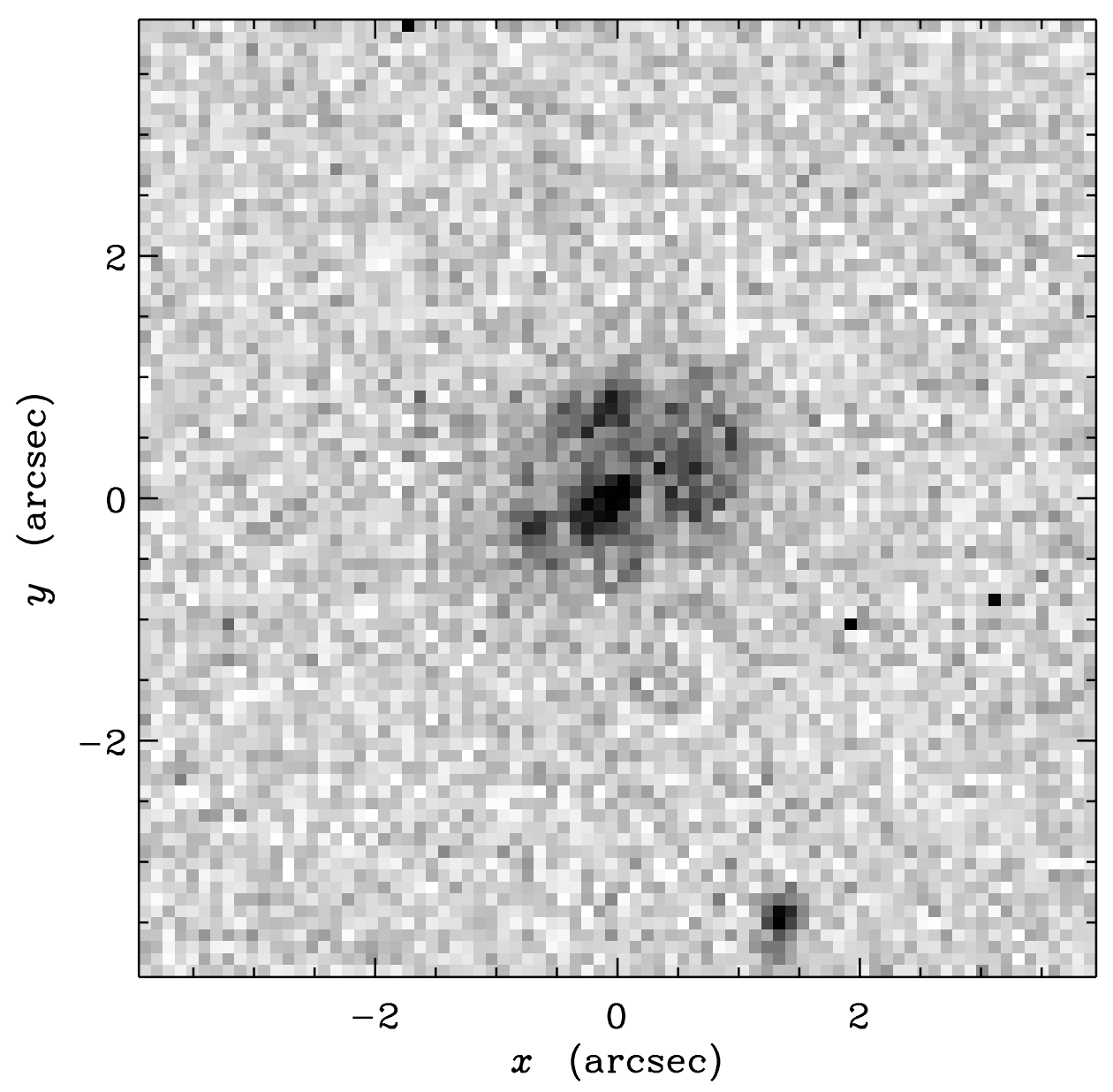

FIG. 5.-F36336_1005: This $z=1.015$ object might either be a distant Ir galaxy or a multicomponent merger. 


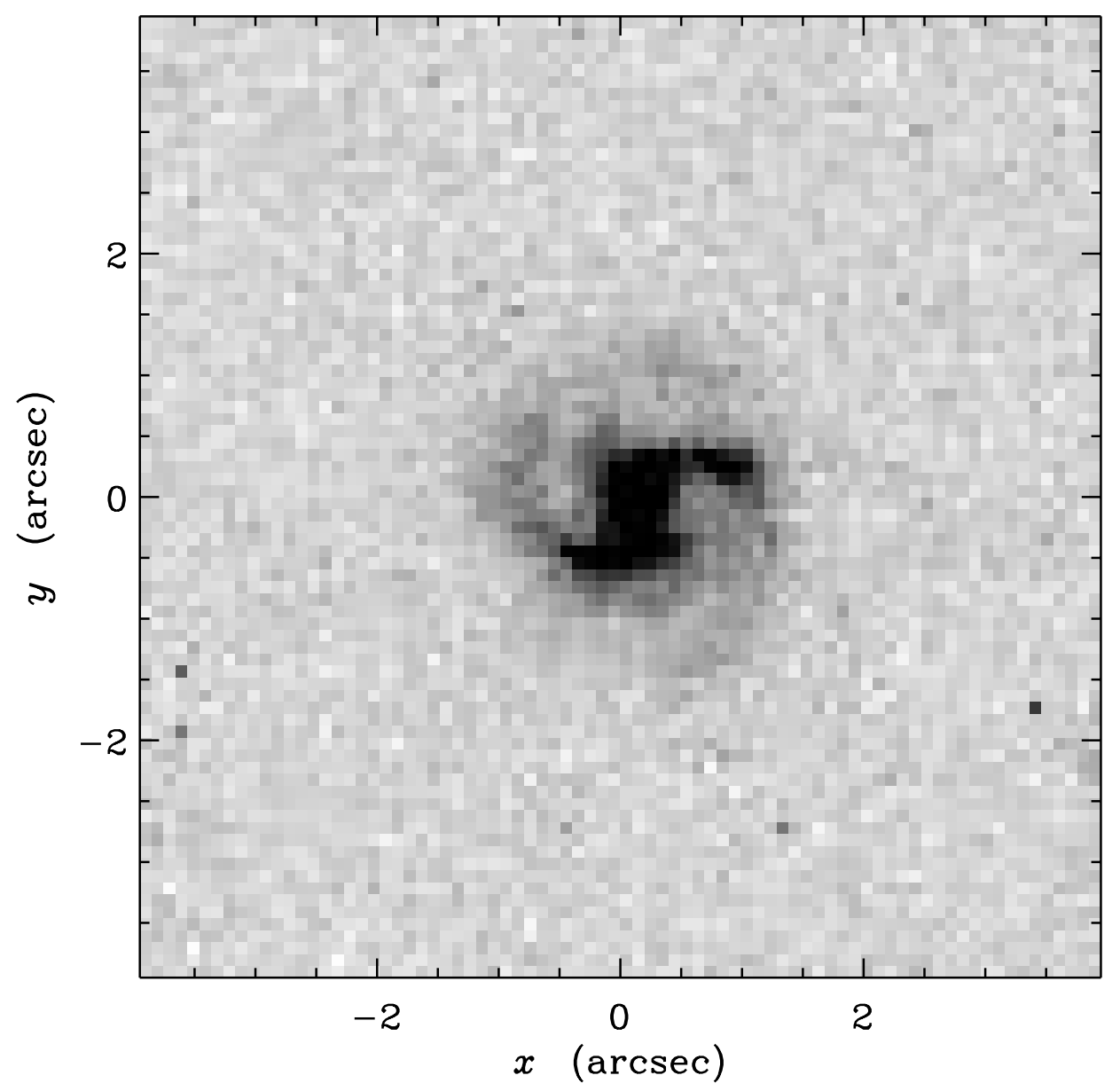

FIG. 6.-F37058_1153: Rare example of a two-armed spiral viewed at a large look-back time $(z=0.904)$.

were inspected, excepting those in which there were serious crowding or edge effects. The classifications for the three redshift intervals, along with commentary, are presented in Tables $1-3$. The $R$ magnitudes from Hogg et al. (2000), the redshifts from Cohen et al. (2000), and the rest-frame $B$ luminosities from Cohen (2000) are also given. See Cohen (2000) for the definition of the rest-frame luminosity and how it is derived.

The fractions of galaxies by type are collected in Table 4 (intermediate types, such as Ir/merger, are counted as $0.5 \mathrm{Ir}$ and 0.5 merger). In the present paper, we have, following van den Bergh et al. (1996), used the somewhat judgmental term "protogalaxy" to denote objects that resemble the prototypical galaxy H36555_1249, which is shown in Figure 10. A color image of this object, which is shown as Figure 5 (Plate 9) of van den Bergh et al. (1996), shows what appears to be a reddish off-center nuclear bulge that is embedded in a rather chaotic-looking disk that contains half a dozen bright blue knots. Objects with this type of morphology were regarded as spirals that are still in the process of being assembled. In objects described as "mergers," the individual components/knots have not yet combined to form a single coherent structure.

\section{GALAXY MORPHOLOGY AT HIGH AND LOW REDSHIFT}

As explained in $\S 1$, we interpret distant galaxies by reference to their local counterparts, as exemplified by the Shapley-Ames galaxies (van den Bergh 1960c, Table 4). The differences are striking.

\subsection{Spirals}

The present sample of galaxies shows an almost complete absence of "grand design" spirals, i.e., disk systems with well-developed long arms of DDO types Sb I, Sbc I, and Sc I (cf. van den Bergh et al. 1996). Previous experience (van den Bergh 1989), which was confirmed by radial velocity observations (Visvanathan \& van den Bergh 1992), showed that such luminous grand-design spirals could have been recognized on images that contain as few as $\sim 100$ silver grains.

Even less pronounced spiral structure, such as is seen in nearby spirals of DDO luminosity classes II, III, and IV, appears to be rare in distant HDF + FF galaxies. Furthermore, the spiral structure that is observed in such distant spirals appears to be more chaotic (i.e., less regular) than do $\mathrm{Sc}$ and Scd spirals at $z \sim 0$. The fraction of Sc and Sc/Ir galaxies increases from $\sim 5 \%$ at $z \sim 1$ to $\sim 10 \%$ at $z \sim 0.5$ and $\sim 23 \%$ at $z \sim 0$. Most of the missing Sc, Scd, and Sc/Ir galaxies at $z \sim 1$ are probably masquerading under the categories "protogalaxy," "peculiar," or "merger." In intermediate- and early-type "spirals," little (or no) evidence is actually seen for spiral arms. At $z \sim 0.8$ this effect might be partly (but not entirely) due to the low spatial resolution with which galaxies at such large redshifts are viewed. However, at $z \sim 0.4$ the almost complete absence of well-developed spiral arms is certainly real. This is so because the mean luminosity of the galaxies in the present sample increases with redshift. Since the strength of spiral structure increases with luminosity, one would actually 


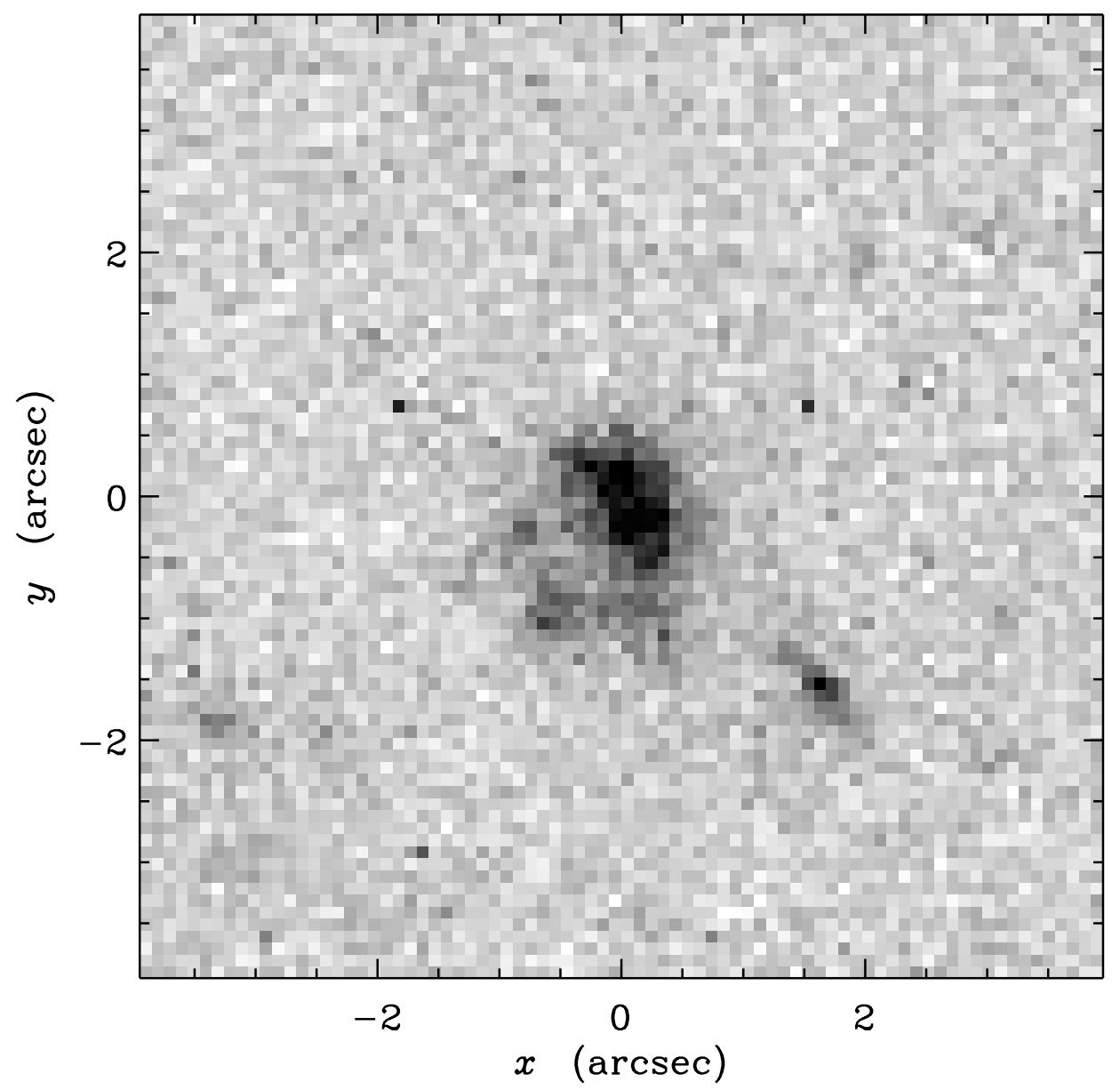

FIG. 7.-F37167_1042: Example (at $z=0.821$ ) of a possible multicomponent merger. Note "bits and pieces" of other, probably more distant, objects in this field.

have expected the strength of spiral arms to increase with $z$. In view of the fact that little or no spiral structure is actually seen at $z \gtrsim 0.5$, the $\mathrm{Sa}, \mathrm{Sb}$, and $\mathrm{Sc}$ classifications listed in Tables 2 and 3 are almost entirely based on the central concentration of light in the galaxy images. In other words, spiral-arm tilt and resolution could usually not be factored into the present classifications of the most distant early-type and intermediate-type spirals.

\subsection{Barred Spirals}

Another striking feature of the present $\mathrm{HDF}+\mathrm{FF}$ sample is the almost complete absence of barred spirals at high redshift. The highest redshift galaxy that almost certainly has a real bar is at $z=0.321$, although two possible barred galaxies in Table 1 have redshifts $z=0.457$ and $z=0.475$, and one from Table 3 has $z=0.962 .{ }^{9}$ Taken at face value, this result suggests that only $\lesssim 1 \%$ of all highredshift galaxies are barred spirals, compared with $21 \%$ to $34 \%$ among nearby spirals (van den Bergh 1998, p. 43).

\subsection{Elliptical Galaxies}

Roughly one-fifth of the galaxies with $z \sim 1$ are compact and have morphologies similar to those of nearby E, S0,

\footnotetext{
${ }^{9}$ Bunker et al. (2000) claim that this object (H36483_1214) seems to be a "chance alignment of a swath of young stars with the approximate axis of the true bar."
}

and Sa galaxies. The data in Table 4 show no statistically significant variation with redshift in the fraction of field galaxies with classification types $\mathrm{E}, \mathrm{E} / \mathrm{S} 0$, and $\mathrm{S} 0$.

It is interesting to note evidence from the morphological classification for at least one density enhancement along the line of sight to the HDF, which contains a clump of nine E0-E3 galaxies with $\langle z\rangle=0.679$. This is one of the most prominent peaks in the redshift distribution of galaxies in the region of the HDF and was noticed as such in the initial redshift survey analysis of Cohen et al. (1996).

\subsection{Peculiar/Merging Galaxies}

Distant galaxies are far more likely to be classified as peculiar. Van den Bergh (1960c) found only 31 out of 540 $(5.7 \%)$ of all nearby spirals of types $\mathrm{Sa}, \mathrm{Sb}$, and $\mathrm{Sc}$ in the Shapley-Ames catalog to be peculiar. After excluding edge-on objects (in which it is difficult to detect any peculiarities), one finds that 17.5 out of $20.5(85 \%)$ of all spirals with types Sa-Sab-Sb-Sbc-Sc and redshifts of $0.25<z<0.60$ are noted as being peculiar in Table 1. Only four out of seven objects of types Sa-Sab are peculiar, whereas all spirals of types $\mathrm{Sb}-\mathrm{Sbc}-\mathrm{Sc}$ are found to be peculiar. Perhaps surprisingly, Table 4 shows that the fraction of peculiar galaxies appears to remain approximately constant over the range $0.25<z<1.2$. The reason for this is, no doubt, that it is much easier to see peculiarities in the relatively large images of nearby galaxies at $z \sim 0.4$ than it is to 


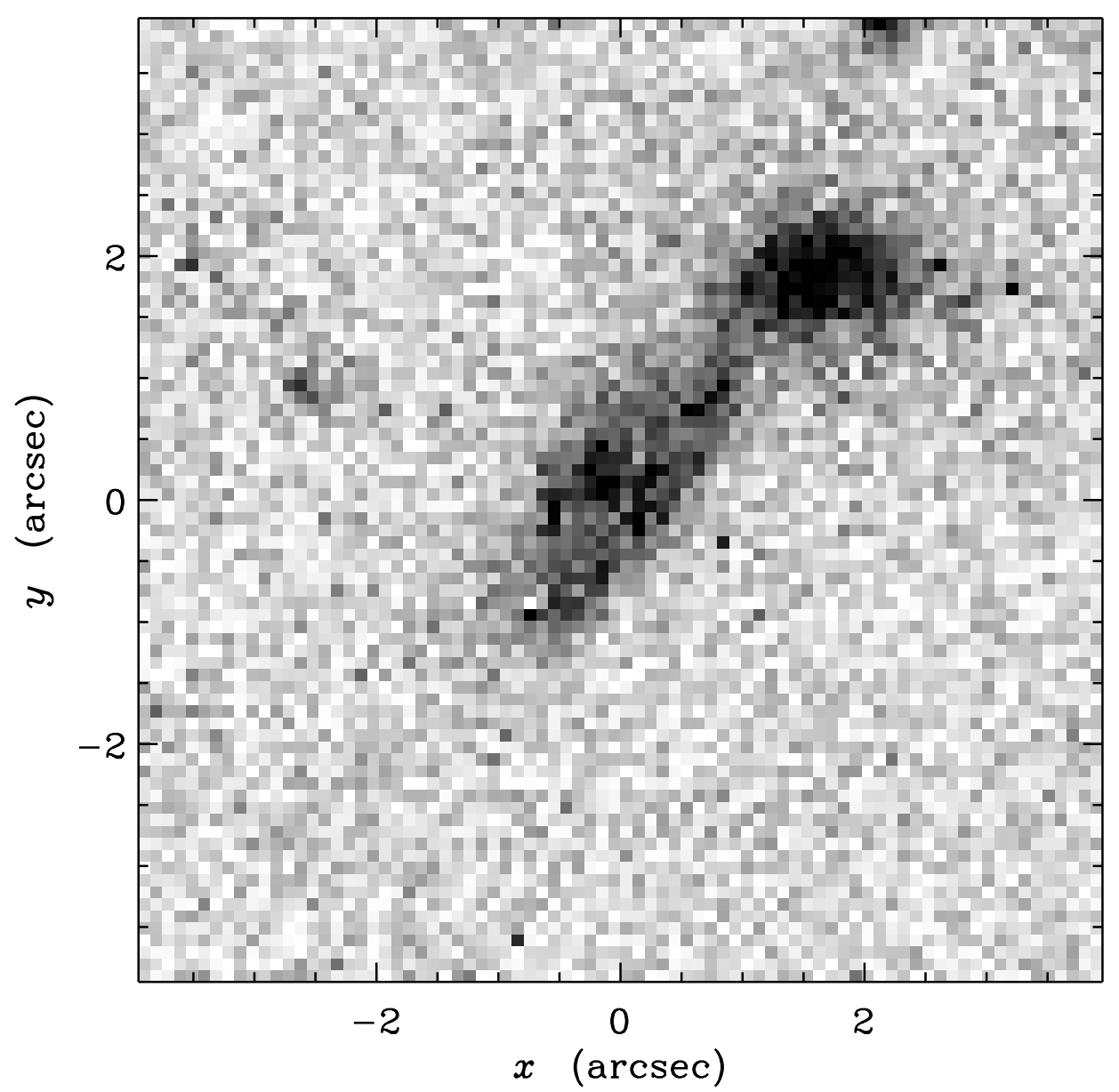

FIG. 8.-F37221_1210: Central part of a "debris field" (with $z=0.928$ ) that may eventually merge into one or more larger galaxies.

notice similar peculiarities in the much smaller images of very distant galaxies with $z \sim 1$.

Similarly, galaxies classified as probable mergers, which account for only $\sim 1 \%$ of the galaxies at $z \sim 0$, represent $\sim 6 \%$ of those with $0.25<z<0.80$ and $14 \%$ at $z \sim 1$. In particular, there is a rapid increase with redshift in the fraction of triple and multiply interacting galaxies. In fact, some small regions of the HDF could be described as debris fields filled with fragments that might eventually merge into one or more major galaxies.

\section{DISCUSSION}

Butcher \& Oemler (1978) first established that cluster galaxies evolve with time. The observations presented here (and in earlier studies cited above) firmly establish that the morphology of field galaxies also evolves over time. The challenge now is to use these empirical observations to infer a physical description of galaxy formation and evolution. (This approach is quite complementary to deductive methods that use "semianalytic" extensions to gravitational instability theory to make quantitative comparisons with measured properties of galaxies.) The most natural way to address this problem is to take the galaxies we see around us today and to ask, in a statistical fashion, what are their histories. However, this will almost certainly lead to an incomplete view because galaxies interact and merge and are otherwise strongly affected by their environment. In addition, many galaxies may become too dim to be observable locally.

\subsection{Spirals}

The low fraction of Sc and Sc/Ir galaxies at high redshift and the almost complete absence of luminous, "grand design" galaxies beyond $z \sim 0.3$, when the universe had an age of $\sim 10 \mathrm{Gyr}$, suggest that a major fraction of the future Sc galaxies are still classified as protogalaxies or mergers at $z \sim 1(t \sim 8 \mathrm{Gyr})$. In other words, many Sc galaxies had not yet fully assembled at this time. This is supported by the observation that most early-type spirals in the field already appear to have achieved a more or less "normal" morphology by $z \sim 0.4$, while late-type spirals still look peculiar. However, it should be emphasized that the individual bits and pieces from which Sc galaxies were assembled might contain quite old stars and clusters.

This deduction, in conjunction with the fact that significant numbers of compact early-type galaxies are observed at high redshifts, may support the view of Boissier \& Prantzos (2000) that the timescale for the formation of lowmass disks is longer than that for more massive ones. This, in turn, is consistent with the view (Bell \& de Jong 2000) that it is the surface density of a young galaxy that drives its rate of star formation. As Boissier \& Prantzos point out, this interpretation may present some difficulties for the standard picture of hierarchical cosmology.

The observation that spiral structure is often poorly developed in galaxies with $z>0.5$ is perhaps not surprising. The rotational period in the outer parts of a giant galaxy ranges from $\sim 0.3$ to $1 \mathrm{Gyr}$, and density wave theory requires several rotational periods for the arms to develop 


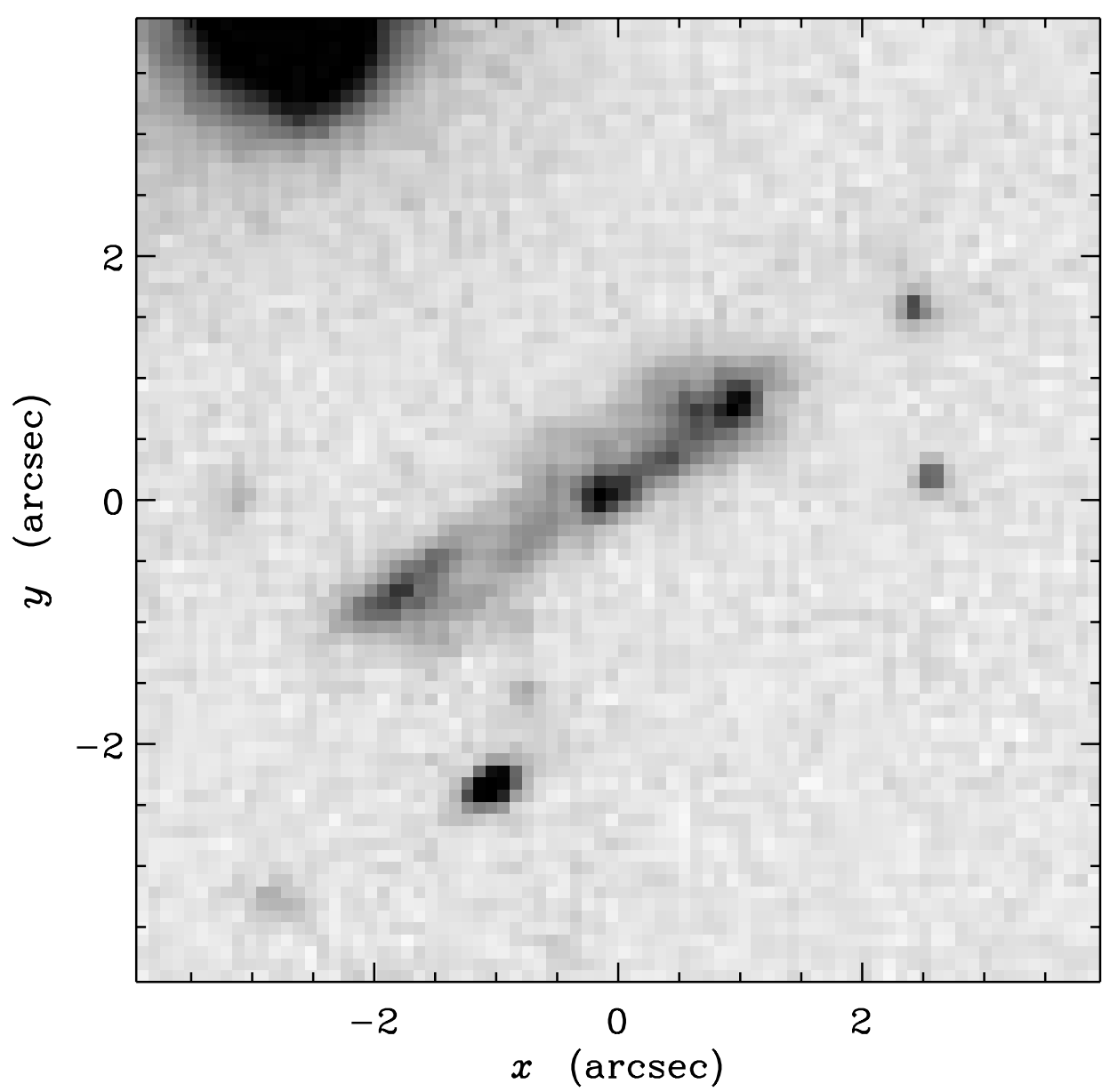

FIG. 9.-H36444_1142: Peculiar edge-on disk or barlike object at $z=1.020$ containing at least three merging components.

fully. Even if a disk forms when the universe is, say, $2 \mathrm{Gyr}$ old, there may be only time for about five rotational periods before it appears in our high-redshift interval. This constraint is particularly relevant to the grand-design spirals, which may not assemble as disks till the universe is $\gtrsim 5 \mathrm{Gyr}$ old and then take another $\sim 5 \mathrm{Gyr}$ to develop their arms. In this connection, it is of interest to note (Fasano et al. 2000) that the galactic population of rich clusters changed significantly even more recently, with spirals being transformed into S0 galaxies between $z \sim 0.1$ and $z \sim 0.25$. Such a transformation of spirals into S0 galaxies might have been produced by ram pressure stripping (Gunn \& Gott 1972) or by galaxy harassment and tidal effects (Abadi, Moore, \& Bower 1999; Quilis, Moore, \& Bower 2000).

\subsection{Barred Spirals}

Our observations suggest that most genuine barred spirals only started to form $\sim 4$ Gyr ago. This may be because galaxies at high $z$ are still too young to have formed dynamically cool disks, which permit barlike instabilities to develop. In this connection it is of interest to note that a number of $\mathrm{Sb}$ galaxies at $z \sim 1$ appear to have real bulges (good examples are F36254_1519, F36481_1102, and F37088_1117). It would be important (but difficult) to do accurate photometry of these spirals to determine whether the cores in these galaxies are true $R^{1 / 4}$ bulges $^{10}$ or if they are just the brightest inner parts of exponential disks. If the presence of true bulges at $z \sim 1$ were to be confirmed, this would militate against the hypothesis of Raha et al. (1991) and Pfenniger, Martinet, \& Combes (1996) that some bulges might have been formed from bars.

\subsection{Elliptical Galaxies}

This study does not shed much light on the evolution of elliptical galaxies, which appear to have mostly formed by $z \sim 1$. The major uncertainty is whether the compact galaxies that are classified as $\mathrm{E}, \mathrm{S} 0$, or Sa at $z \sim 1$ are really bulges or if we are already observing disks (cf. Marleau \& Simard 1998). This is a crucial test for theories of galaxy formation. However, this issue is complicated by the observation (Graham \& Prieto 1999) that the bulges of many late-type spirals are best described by exponential luminosity profiles.

\subsection{Peculiar/Merging Galaxies}

Among nearby NGC galaxies, perhaps only $\sim 0.1 \%$ are members of multiple interacting/merging systems that

\footnotetext{
${ }^{10}$ Falomo et al. (1997) have previously established that a compact galaxy with $z=0.19$ has an $R^{1 / 4}$ profile.
} 


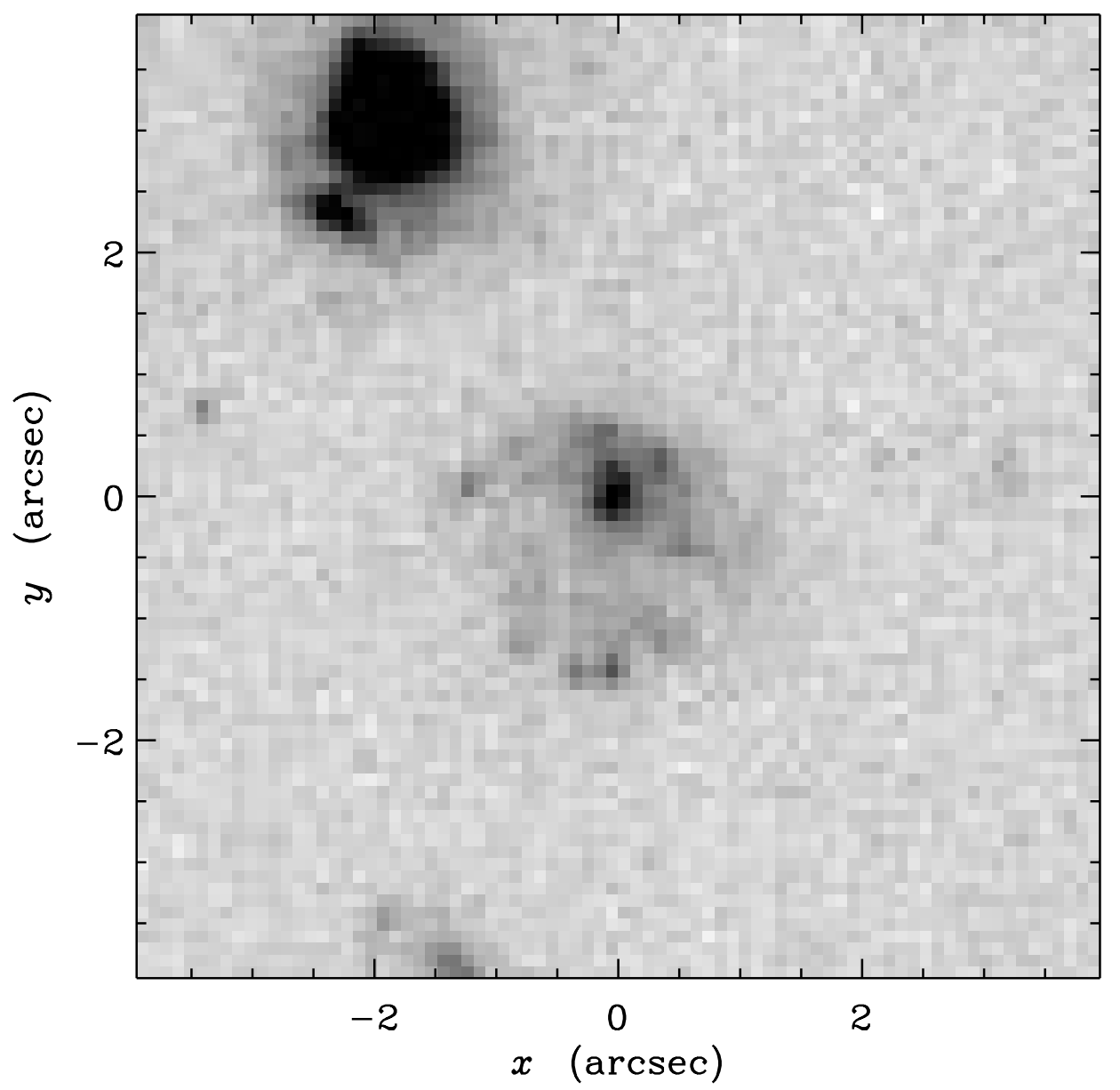

FIG. 10.-H36555_1249: Probable protospiral at $z=0.950$. Multicolor images show that the dense core of this object is red (old) and that the outer knots are blue (young).

resemble Stefan's Quintet (NGC 7317-7319). On the other hand, a significant fraction of the present sample of HDF and FF objects appear to be members of (or associated with) multiple merging systems. This result is consistent with the view (Toomre 1977; Abraham 1999; Carlberg et al. 2000; Le Fèvre et al. 2000) that mergers were much more frequent in the past than they are at the present time. However, debate continues (e.g., Carlberg et al. 2000) about the rate at which such mergers increase with redshift, and regarding the mass range of merging galaxies.

An important caveat is that some close pairs may consist of projected superposed images of unrelated galaxies. In all four cases in which the redshifts of both of a pair of galaxies S. v. d. B. considered to be mergers are known, the redshifts are discrepant, and the objects do not appear to be physically associated (see the notes to Tables 1,2 and 3). ${ }^{11}$ This serves again as a warning that, in the absence of measured redshifts for all component galaxies, only obvious tidal distortions should move a "merger suspect" into the "merger" category.

\subsection{Morphological Classification at High Redshift}

Van den Bergh et al. (1996) pointed out that many of the images of very distant HDF and FF galaxies do not fit

\footnotetext{
${ }^{11}$ In one of the four cases, one of the redshifts is uncertain. In the other three cases, Cohen et al. (2000) regard both redshifts as secure.
}

comfortably into the Hubble (1936) classification scheme, which we now find to be only strictly applicable to field galaxies with $z \lesssim 0.3$. In particular, since spiral structure was rare (or absent) prior to this time, the DDO luminosity classification system (van den Bergh 1960a, 1960b, 1960c; Sandage \& Tammann 1981) is not especially useful.

Can we devise a galaxy classification scheme that would have been useful to sapient beings who might have been surveying galaxies in their neighborhood some $5 \mathrm{Gyr}$ ago? The search for such a system is made more difficult by the unfortunate fact that it is often not clear whether adjacent luminous clumps should be regarded as separate galaxies, or as condensations within a single, larger protogalaxy. In fact, such a dichotomy is not even physically significant if initially separate ancestral bits and pieces eventually merge into a single galaxy. Possibly, a two-parameter classification system based on central concentration of light (Morgan 1958, 1959) and asymmetry (Abraham et al. 1996b) or clumpiness, perhaps supplemented by color information, may represent the best that can be done regarding the classification of galaxies in the early universe. In rich nearby clusters, only the central concentration of light appears to be a useful classification parameter.

In this study we have used redshift data and the flanking field images to substantiate many of the original reactions to the first inspection of the Hubble Deep Field over 4 years ago. In addition we have shown that the development of spiral structure, both wound and barred, is delayed until the 
universe is typically $\sim 10 \mathrm{Gyr}$ old. Perhaps the most pressing current task is to determine whether the compact objects observed at $z \sim 1$ are disks or spheroids. Overall, though, we remain impressed by the strong evolution in galaxy morphology. As Hartley (1953) wrote in The GoBetween, "The past is a foreign country; they do things differently there."

It is a pleasure to thank Peter Stetson for his help with the rescaling of the present images, Simon Morris for cosmological consultations, and Jerry Sellwood for information on the formation of galactic bars. The extragalactic work of J. G. C., who is the principal investigator of the Caltech Faint Galaxy Redshift Survey, is not supported by any federal agency. R. B. acknowledges support under NSF grant AST 99-00866.

\section{REFERENCES}

Abadi, M. G., Moore, B., \& Bower, R. G. 1999, MNRAS, 308, 947

Abraham, R. G. 1999, in IAU Symp. 186, Galaxy Interactions at Low and High Redshift, ed. J. E. Barnes \& D. B. Sanders (Dordrecht: Kluwer), 11

. 2000, in Toward a New Millennium in Galaxy Morphology, ed. D. L. Block, I. Puerari, A. Stockton, \& D. Ferreira (Dordrecht: Kluwer), 323

Abraham, R. G., Ellis, R. S., Fabian, A. C., Tanvir, N. R., \& Glazebrook, K. 1999a, MNRAS, 303, 641

Abraham, R. G., Merrifield, M. R., Ellis, R. S., Tanvir, N. R., \& Brinchmann, J. 1999b, MNRAS, 308, 569

Abraham, R. G., Tanvir, N. R., Santiago, B. X., Ellis, R. S., Glazebrook, K., \& van den Bergh, S. 1996a, MNRAS, 279, L47

Abraham, R. G., van den Bergh, S., Glazebrook, K., Ellis, R. S., Santiago, B. X., Surma, P., \& Griffiths, R. E. 1996b, ApJS, 107, 1

Amis, M. 1997, interview by J. Cowley, Times (London), August 21, Features section

Bell, E. F. \& de Jong, R. S. 2000, MNRAS, 312, 497

Boissier, S., \& Prantzos, N. 2000, MNRAS, 312, 398

Brinchmann, J., et al. 1998, ApJ, 499, 112

Bunker, A., Spinrad, H., Stern, D., Thompson, R., Moustakas, L., Davis, M., \& Dey, A. 2000, in Lecture Notes in Physics, Galaxies in the Young Universe II, ed. H. Hippelein \& K. Meisenheimer (Berlin: Springer), in press (astro-ph/0004348)

Butcher, H., \& Oemler, A., Jr. 1978, ApJ, 219, 18

Carlberg, R. G., et al. 2000, ApJ, 532, L1

Cohen, J. G. 2000, AJ, submitted

Cohen, J. G., Cowie, L. L., Hogg, D. W., Songaila, A., Blandford, R., Hu, E. M., \& Shopbell, P. 1996, ApJ, 471, L5

Cohen, J. G., Hogg, D. W., Blandford, R. D., Cowie, L. L., Hu, E., Songaila, A., Shopbell, P., \& Richberg, K. 2000, ApJ, 538, 29

Corbin, M. R., Vacca, W. D., O'Neil, E., Thompson, R. I., Rieke, M. J., \& Schneider, G. 2000, AJ, 119, 1062

Cowie, L. L., Hu, E. M., \& Songaila, A. 1995, AJ, 110, 1576

Dressler, A. 1980, ApJ, 236, 351

Driver, S. P., Fernández-Soto, A., Couch, W. J., Odewahn, S. C., Windhorst, R. A., Phillipps, S., Lanzetta, K., \& Yahil, A. 1998, ApJ, 496, L93

Eskridge, P. B., et al. 2000, AJ, 119, 536

Falomo, R., Urry, C. M., Pesce, J. E., Scarpa, R., Giavalisco, M., \& Treves, A. 1997, ApJ, 476, 113
Fasano, G., Poggianti, B. M., Couch, W. J., Bettoni, D., Kjærgaard, P., \& Moles, M. 2000, ApJ, in press

Ferguson, H. C., Dickinson, M., \& Williams, R. 2000, ARA\&A, in press

Graham, A. W., \& Prieto, M. 1999, ApJ, 524, L23

Gunn, J. E., \& Gott, J. R., III. 1972, ApJ, 176, 1

Hartley, L. P. 1953, The Go-Between (London: H. Hamilton)

Hogg, D. W., et al. 2000, ApJS, 127, 1

Hubble, E. P. 1936, The Realm of the Nebulae (New Haven: Yale Univ. Press)

Le Fèvre, O., et al. 2000, MNRAS, 311, 565

Lilly, S., et al. 1998, ApJ, 500, 75

Marleau, F. R., \& Simard, L. 1998, ApJ, 507, 585

Morgan, W. W. 1958, PASP, 70, 364 1959, PASP, 71, 394

Odewahn, S. C., Windhorst, R. A., Driver, S. P., \& Keel, W. C. 1996, ApJ, 472, L13

Pfenniger, D., Martinet, L., \& Combes, F. 1996, in New Extragalactic Perspectives in the New South Africa, ed. D. L. Block \& J. M. Greenberg (Dordrecht: Kluwer), 291

Quilis, V., Moore, B., \& Bower, R. 2000, Science, 288, 1617

Raha, N., Sellwood, J. A., James, R. A., \& Kahn, F. D. 1991, Nature, 352, 411

Sandage, A., \& Tammann, G. A. 1981, A Revised Shapley-Ames Catalog of Bright Galaxies (Washington: Carnegie Inst. Washington) (RSA)

Schade, D., et al. 1999, ApJ, 525, 31

Simard, L., et al. 1999, ApJ, 519, 563

Toomre, A. 1977, in The Evolution of Galaxies and Stellar Populations, ed.

B. M. Tinsley \& R. B. Larson (New Haven: Yale Univ. Obs.), 20

van den Bergh, S. 1960a, ApJ, 131, 215

-1960b, ApJ, 131, 558

. 1960c, Publ. David Dunlap Obs., 2, 159 . 1989, AJ, 97, 1556

. 1998, Galaxy Morphology and Classification (Cambridge: Cambridge Univ. Press)

van den Bergh, S., Abraham, R. G., Ellis, R. S., Tanvir, N. R., Santiago, B. X., \& Glazebrook, K. G. 1996, AJ, 112, 359

van den Bergh, S., \& Pierce, M. J. 1990, ApJ, 364, 444

Visvanathan, N., \& van den Bergh, S. 1992, AJ, 103, 1057

Williams, R. E., et al. 1996, AJ, 112, 1335 